\title{
Smart damping of skew composite plates using Murakami zig-zag function
}

\author{
N. Mehadi Khan ${ }^{1} \cdot$ R. Suresh Kumar ${ }^{1}$ (])
}

Received: 27 August 2020 / Accepted: 24 February 2021 / Published online: 20 March 2021

(c) The Author(s) 2021 OPEN

\begin{abstract}
The present work is aimed at deriving a finite element model for active constraining layer damping treatment (ACLD) of layered skew plates by incorporating zig-zag behaviour using a Murakami zig-zag function (MZZF). The ACLD in skew patch form comprises of 1-3 PZC material and viscoelastic material in the layer form placed on substrate skew plate. The overall skew substrate ACLD system deformation kinematics are derived using MZZF and the equations of motion for the same are derived by virtual work method. A MATLAB subroutine for the overall skew plate ACLD system has been developed to present the closed loop frequency responses by successful implementation of closed-loop feedback system. The substrate skew plates with different lamination schemes namely symmetric/antisymmetric cross-ply and antisymmetric angle-ply are considered to assess the damping behavior of the skew plates undergoing ACLD. Also, the piezo-fiber angle (obliquely reinforced) variation of the PZC layer on the damping responses of the skew plates have been thoroughly examined.
\end{abstract}

Keywords Finite element method (FE) · Smart damping · Piezoelectric composite (PZC) · Murakami zig-zag function (MZZF) - Skew plates

\section{Introduction}

It is apparent that layered laminates with skew edges are imperative to many of the applications and have substantial importance in aerospace systems, frames, ship hulls, and aircraft wings. These elements exhibit anisotropic behavior due to discontinuities at layer interfaces. Also, the low transverse to in-plane modulus ratio and different transverse moduli results in the zig-zag behavior in the element. Hence the behavioural study of skew elements is an important area to be dealt with and investigations in this regard has be carried out by few researchers in the past decades. Wang [1] applied Reissner-Mindlin model considering the transverse shear effect and studied the free vibration responses of skew laminates. Krishna and Palaninathan [2] developed triangular element for finite element (FE) model for free vibration bending behavior of skew plates. First order shear deformation theory (FSDT) and higher order shear deformation theory (HSDT) are used to analyse the buckling of skew panels by Babu and Kant [3]. Garg et. al [4] presented a simple $\mathbf{C}^{\mathbf{0}} \mathbf{F E}$ model based on HSDT for free vibration study of composite and skew sandwich plates. Recently Kiani [5] explore the buckling behaviour of functionally graded carbon nanotube (FG-CNT) reinforced skew plates and evaluated the temperature dependent responses of the same. Most recently, Kallannavar et al. [6] studied the free vibration characteristics of skew composite and sandwich plates and reported the hygrothermal effect on the same. But the analysis using these theories is time consuming leading to high costs of computation. Also, these theories do not account for the Zig-Zag effect which may affect the responses

R. Suresh Kumar, rskumar.me@nitrr.ac.in | 'Department of Mechanical Engineering, National Institute of Technology Raipur, Raipur, Chhattisgarh 492010, India. 
of layered structures. Hence, Murakami [7] modified the existing theories by introducing Murakami zig-zag function (MZZF) to account for this behavior. MZZF incorporation considers use of fewer degrees of freedom (DOF) and hence the low computation time and cost. Carrera [8] introduced the MZZF modifying the classical theories investigating the effect in modeling the plates and shells. Carrera and Ciuffreda [9] developed a unified theory for assessing the behaviour of layered plates under bending. Brischetto et al. [10] used the MZZF for the analysis of unsymmetrical bending of sandwich laminates. Brischetto et al. [11] employed the incorporated MZZF higher order theory for studying the bending responses of panels of sandwich construction.

These structures possess very low damping which may result in vibration related failures of the same. To increase damping these structures are often embedded with additional materials to enhance their damping and once such material which is widely used is the piezoelectric material. Such structures with embedded piezoelectric type material are called smart structures [12]. Piezoelectric material embedded flexible structures have been studied rigorously by many researchers. Bailey and Hubbard [12] analysed the transverse vibration control of the laminated beam by the use of polymer made of piezo material. Reddy [13] first used the piezo electric materials as sensor and actuator for carrying out the analysis of laminated plates. Recently Elahi et al. [14] proposed a Barium titanate piezoelectric material for aerospace applications and evaluated its performance experimentally for thermo mechanical shock and electrical shock. These materials require very high voltages for producing the desired effect and hence usage of these materials in bulk form posed a huge setback. Hence, these materials are reinforced in polymer acting as matrix lead to the development of piezoelectric composite (PZC). Initial research in this area is carried of effective properties estimation of these PZCs. Smith and Auld [15] have done the pioneering work associated with the modeling of 1-3 PZC material for studying the bending oscillations of structures. Dunn and Taya [16] employed micromechanics approaches to estimate the moduli of the PZCs. Stevenson et al. [17] proposed piezoelectric materials for high temperature applications for their possible use as sensors and actuators. Most Recently, Wang et al. [18] conducted the survey of the piezoelectric material and their use in recent years and based on their findings they made classifications of the same depending on the long working strokes. Among the class of PZCs, 1-3 PZC [19] materials having vertical and oblique piezo fiber reinforcements are widely used for damping of thin structures. To enhance the damping responses, 1-3 PZC material along with viscoelastic material are embedded over the structure where the later acts as passive material while the former acts as active material when supplied with voltage. Such combo of 1-3 PZC and the viscoelastic material developed by Baz [20] who coined that as active constrained layer damping (ACLD). Baz and Ro [21] optimized the damping and energy dissipation of plates undergoing ACLD treatment and proved that damping performance is enhanced by the use of ACLD over just 1-3 PZC layer. ACLD treatment is nothing but a patch of material consisting of a viscoelastic material as constrained layer and a smart material layer like the piezoelectric material layer as constraining layer. Both active (with supply voltage) and passive (with no voltage) vibration control can be achieved by using the ACLD treatment [22]. Recently, several authors examined the behavior of 1-3 PZC as a material of the ACLD constraining layer for damping the responses of composite/ sandwich plates and shells. Kanasogi and Ray [23], presented ACLD of skew plates composed of laminates considering the displacement equations in terms of FSDT. Selim et al. [24] carried out the vibration control of functionally graded material (FGM) plates using HSDT. Zhao et al. [25] presented the damping behaviour considering an equivalent single-layer material by simplifying the finite element model of the plates. Selim et al. [26] reported the control of FG graphene nanoplatelets reinforced plates using HSDT with integrated piezoelectric layers. Recently, Li et al. [27] investigated the damping response of FG piezoelectric plates composed of smart piezo layers using the Hamiltonian and Rayleigh ritz mechanics. Vinyas [28] carried out the vibration damping analysis of skew magneto electro-elastic plates using FSDT. Most of the above-mentioned studies considered the FSDT and HSDT to model the displacement kinematics in deriving the governing equations for suppressing the vibration. However, no work has yet been reported with the inclusion of the MZZF which play a vital role owing to the anisotropic behaviour of these structures and also resulting in an effective computational model for the analysis of structures undergoing ACLD. Very recently, Khan and Kumar [29] incorporated the MZZF for reporting the smart damping analysis of cylindrical shells. The findings reported by them prove the effectiveness of the model in reducing the computation time. Although MZZF has been successfully implemented in studying the behaviour of cylindrical shells, this provides a scope for investigating the effect of the same in the analysis of laminated skew plates. Also, the response and damping characteristics of skew plates are entirely different compared to the cylindrical shells and hence it provides an ample scope for investigating them. The novelty of the present work lies in the successful incorporation of $\mathbf{M Z Z F}$ for estimating the vibration damping of smart skew plates. In the present paper, the authors intend to develop a closed loop finite element (FE) model considering the displacement kinematics incorporating the $\mathbf{M Z Z F}$ 
to examine the damping responses of ACLD smart skew laminate plate. A MATLAB subroutine has been developed to investigate the damping behaviour of plates in both active $($ voltage $\neq \mathbf{0})$ and passive $($ voltage $=\mathbf{0})$ mode.

\subsection{Outlines of the paper}

The present article is organised systematically in the following manner:

- First, the governing equations have been derived including the MZZF in the displacement functions. The stresses and strains at any point in the skew plate have been obtained by the use of strain displacement relations while the equations of motion of the skew plate are derived employing the virtual work principle.

- The finite element model to obtain elemental, global equations of motion are obtained for which eightnoded isoparametric elements have been considered.

- Employing a basic feed-back control strategy, the closed loop global equations of motion are derived for producing the frequency responses in active and passive mode.

- The closed loop global equations of motion have been used to produce the numerical results which are illustrated in the results and discussions part of the manuscript.

- Finally, the conclusions of the study are presented at the end followed by the references section.

\section{Governing equations}

Figure 1 displays skew plate ACLD system composed of $\boldsymbol{N}$ layered substrate laminate with equal lateral dimension namely the length, the width $\mathbf{a}, \mathbf{b}(\mathbf{a}=\mathbf{b})$ while the thickness of the skew substrate plate is $\boldsymbol{h}$. Skew patches of ACLD two in number are positioned on top of substrate skew plate along the non-skewed edges. The smartness adding material of the skewed ACLD is either vertically/ obliquely 1-3 PZC while the passive material is viscoelastic whose thicknesses are $\boldsymbol{h}_{\boldsymbol{p}}$ and $\boldsymbol{h}_{\boldsymbol{v}}$, respectively. The skew angle of the edge of ACLD plate system is $\boldsymbol{\alpha}$ whose reference plane $(\boldsymbol{z}=0)$ overlaps with the mid layer of the substrate plate and the origin of which is located at the left edge of the intersection of the boundary lines namely $\boldsymbol{x}=(\mathbf{y} \tan \boldsymbol{\theta}, \mathbf{a}+\mathbf{y} \tan \theta)$ and $\boldsymbol{y}=(0, \mathbf{b} \cos \theta)$. The orientation $\boldsymbol{\theta}$ of the fibers in the substrate lamina are in the $\boldsymbol{x y}$-plane. The 1-3 PZC smart material with fiber reinforcements in $\mathbf{x z}$-or $\mathbf{y z}$-plane while the same are at angle $\boldsymbol{\psi}$ with $\mathbf{z}$-axis for oblique reinforcements and such depiction pictorially is shown in Fig. 2. The skew ACLD plate is numbered by $\boldsymbol{k}(=1,2,3 \ldots \boldsymbol{N}, \boldsymbol{N}+1, \boldsymbol{N}+2)$ with $\boldsymbol{k}=(1,2,3 \ldots \boldsymbol{N})$ links to the $\boldsymbol{N}$ layered skew substrate while $\boldsymbol{k}=\boldsymbol{N}+1, \boldsymbol{N}+2$ designate the two layers of the ACLD skew patch. A layer with thickness $\boldsymbol{h}^{\boldsymbol{k}}$ is depicted by its top and bottom surface by thickness coordinate given by $\boldsymbol{z}_{\boldsymbol{k}+1}$ and $\boldsymbol{z}_{\boldsymbol{k}}$ with origin corresponds to midplane by the consideration of non-dimensional coordinate $\zeta_{k}$ (function of z) whose limits are $-1 \leq \zeta_{\boldsymbol{k}} \leq 1$. The $\mathbf{M Z Z F}$ in terms of $\zeta_{\boldsymbol{k}}$ is given by the following equation

$M(z)=(-1)^{k} \zeta_{k}$ where $\zeta_{k}=\frac{2}{h_{k}}\left(z-\frac{1}{2}\left(z_{k}+z_{k+1}\right)\right)$

where $M(z)$ is a linear piecewise function in $\boldsymbol{z}_{\boldsymbol{k}}$ assuming values -1 and +1 alternatively at the layer interface. The amplitude of $M(z)$ is independent of both the thickness and the properties of the layer while its derivative $M^{\prime}(z)=d M / d z$ assumes opposite sign with the amplitude dependent only on the layer thickness. To incorporate ZigZag behaviour in the present analysis, the displacement equations in $\boldsymbol{x}$ - and $\boldsymbol{y}$-directions are included with MZZF whose deformation kinematics are demonstrated in Fig. 3. The displacements of skew plate ACLD system in $\boldsymbol{x}$-and $\boldsymbol{y}$-directions namely $\boldsymbol{u}^{\boldsymbol{k}}$ and $\boldsymbol{v}^{\boldsymbol{k}}$ at any point are given by
Fig. 1 Representation of ACLD Skew substrate plate composed of layered laminates

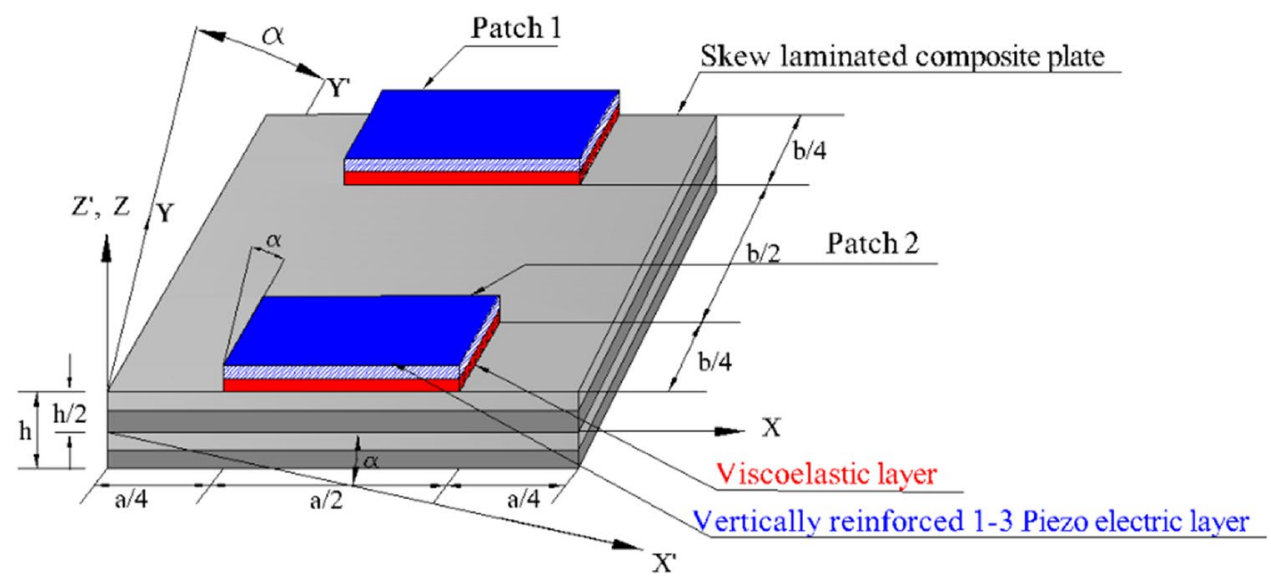




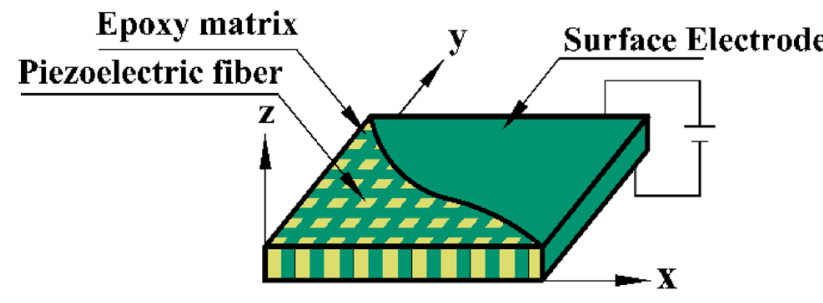

(a)

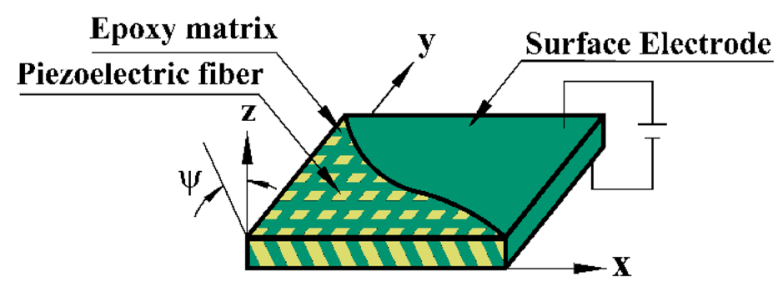

(b)

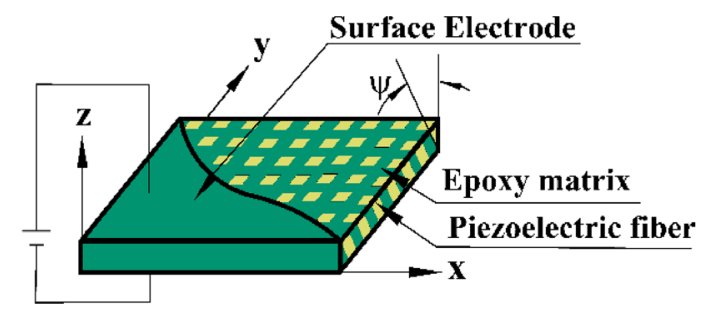

(c)

Fig. 2 Lamina of $1-3$ PZCS with obliquely oriented fibers $(\boldsymbol{\psi} \neq 0)$ when fibers are coplanar with [29] (a) $\boldsymbol{x z}$-plane, (b) $\boldsymbol{y z}$-plane and (c) $\boldsymbol{\psi}=0$ with $\boldsymbol{x z}$-plane (vertical fibers)

$u^{k}=u_{0}+\left(z_{1}^{k}+z_{2}^{k}+z_{3}^{k}\right) \times \theta_{x}+M(z) \times \psi_{x}$

$v^{k}=v_{0}+\left(z_{1}^{k}+z_{2}^{k}+z_{3}^{k}\right) \times \theta_{y}+M(z) \times \psi_{y}$

the variables $\boldsymbol{\Psi}_{\mathrm{x}}$ and $\boldsymbol{\Psi}_{\mathrm{y}}$ are the additional DOF depicting the zig-zag layer wise rotations. With supply of voltage, the patches get activated resulting in the flexure vibration control for which the transverse deformation $\boldsymbol{w}^{\boldsymbol{k}}$ of the skew plate at any point assumed to quadratically vary in $\mathbf{z}$-direction given by:

$\mathrm{w}^{\mathrm{k}}=\mathrm{w}_{0}+\mathrm{z} \times \theta_{\mathrm{z}}+\mathrm{z}^{2} \times \phi_{\mathrm{z}}$

The reference plane displacements along $\boldsymbol{x}, \boldsymbol{y}$ and $\boldsymbol{z}$ -directions are $\boldsymbol{u}_{0}, \boldsymbol{v}_{0}$ and $\mathbf{w}_{0}$ while rotations of the midplane normal of skew plate are $\boldsymbol{\theta}_{\boldsymbol{x}}$ and $\boldsymbol{\theta}_{\boldsymbol{y}}$ in $\mathbf{x z}$-plane, $\boldsymbol{y z}$ -plane, respectively while the rotations depicting gradient and second derivative displacement in $\boldsymbol{z}$-direction are given by $\boldsymbol{\theta}_{\mathbf{z}}$ and $\phi_{\mathbf{z}}$. The right-hand side of the Eqs. (2) to

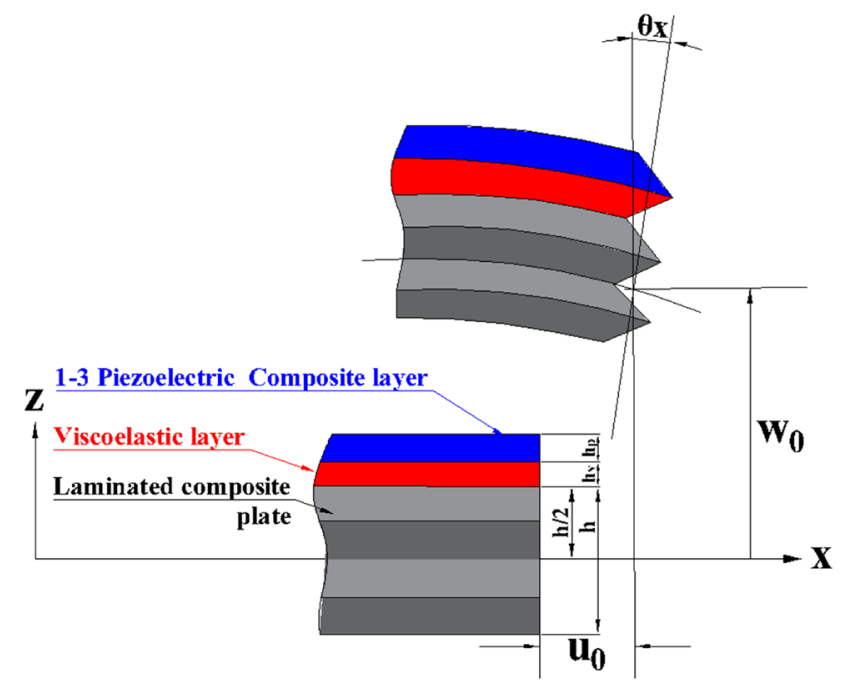

(a)

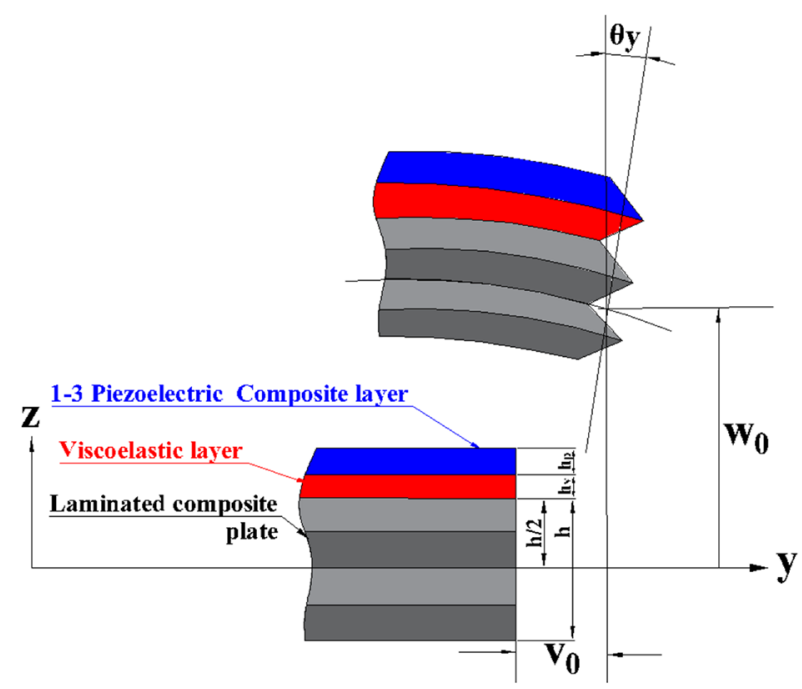

(b)

Fig. 3 Deformation kinematics of the Skew substrate plate ACLD system cross section (a) $\mathbf{x z}$-plane and (b) yz-plane

(4) are functions of $(\mathbf{x}, \mathbf{y}, \mathbf{t})$ while the left-hand side of the same are functions of $(\mathbf{x}, \mathbf{y}, \mathbf{z}, \mathbf{t})$, respectively.

$$
\begin{aligned}
& z_{1}^{k}=z \text { and } z_{2}^{k}=z_{3}^{k}=0 \text { for } k=N \text { (Substrate plate) } \\
& \text { where } \left.\quad z_{1}^{k}=\frac{h}{2}, z_{2}^{k}=\left(z-\frac{h}{2}\right) \text { and } z_{3}^{k}=0 \text { for } k=N+1 \text { (visco }- \text { layer }\right) \\
& z_{1}^{k}=\frac{h}{2}, z_{2}^{k}=h_{v} \text { and } z_{3}^{k}=\left(z-\frac{h}{2}-h_{v}\right) \text { for } k=N+2(P Z C-\text { layer })
\end{aligned}
$$

For maintaining the dimensional equality, variables are grouped as two vectors shown below:

$\left(\left\{d_{t}\right\}\left\{d_{r}\right\}\right)=\left\langle\left[\begin{array}{lll}u_{0} & v_{0} & w_{0}\end{array}\right]^{\top}\left[\theta_{x} \theta_{y} \theta_{z} \Psi_{x} \psi_{y} \phi_{z}\right]^{\top}\right\rangle$ 
Shear locking is evident in thin structures and to avoid this effect, selective integration has be performed using Gauss quadrature rule and hence, the stresses and strains are grouped as in-plane $\left\{\sigma_{b}\right\},\left\{\varepsilon_{b}\right\}$ and out of plane $\left\{\sigma_{s}\right\},\left\{\varepsilon_{s}\right\}$ terms separately and are given as follows.

$$
\begin{aligned}
& \left\{\sigma_{\mathrm{b}}\right\}=\left[\begin{array}{llll}
\sigma_{\mathrm{x}} & \sigma_{\mathrm{y}} & \sigma_{\mathrm{xy}} & \sigma_{\mathrm{z}}
\end{array}\right]^{\top} \text { and }\left\{\varepsilon_{\mathrm{b}}\right\}=\left[\begin{array}{llll}
\varepsilon_{\mathrm{x}} & \varepsilon_{\mathrm{y}} & \varepsilon_{\mathrm{xy}} & \varepsilon_{\mathrm{z}}
\end{array}\right]^{\top} \\
& \left\{\sigma_{s}\right\}=\left[\sigma_{x z} \sigma_{y z}\right]^{\top} \text { and }\left\{\varepsilon_{s}\right\}=\left[\begin{array}{ll}
\varepsilon_{x z} & \varepsilon_{y z}
\end{array}\right]^{\top}
\end{aligned}
$$

in which $\left\{\sigma_{b}\right\},\left\{\varepsilon_{b}\right\}$ feature normal stresses and strains in $\mathbf{x}, \mathbf{y}$ and $\mathbf{z}$-directions along with $\sigma_{x y}$ and $\varepsilon_{x y}$ the in-plane shear stress and corresponding strain while $\left\{\sigma_{s}\right\},\left\{\varepsilon_{s}\right\}$ feature the remaining shear components, respectively. Considering infinitesimal strain analysis, strain displacement relations which are linear have been used for obtaining the strain vectors for skew substrate $\left(\left\{\varepsilon_{b}\right\}_{c^{\prime}}\left\{\varepsilon_{s}\right\}_{c}\right)$, viscoelastic layer $\left(\left\{\varepsilon_{b}\right\}_{v^{\prime}}\left\{\varepsilon_{s}\right\}_{v}\right)$ and 1-3 PZC layer $\left(\left\{\varepsilon_{b}\right\}_{p^{\prime}}\left\{\varepsilon_{s}\right\}_{p}\right)$ which are given by

$$
\begin{aligned}
\left(\left\{\varepsilon_{\mathrm{b}}\right\}_{\mathrm{c}^{\prime}}\left\{\varepsilon_{\mathrm{s}}\right\}_{\mathrm{c}}\right) & =\left(\left\{\varepsilon_{\mathrm{bt}}\right\}+\left[\mathrm{Z}_{1}\right]\left\{\varepsilon_{\mathrm{br}}\right\},\left\{\varepsilon_{\mathrm{st}}\right\}+\left[\mathrm{Z}_{4}\right]\left\{\varepsilon_{\mathrm{sr}}\right\}\right) \\
\left(\left\{\varepsilon_{\mathrm{b}}\right\}_{\mathrm{v}^{\prime}},\left\{\varepsilon_{\mathrm{s}}\right\}_{\mathrm{v}}\right) & =\left(\left\{\varepsilon_{\mathrm{bt}}\right\}+\left[\mathrm{Z}_{2}\right]\left\{\varepsilon_{\mathrm{br}}\right\},\left\{\varepsilon_{\mathrm{st}}\right\}+\left[\mathrm{Z}_{5}\right]\left\{\varepsilon_{\mathrm{sr}}\right\}\right) \text { and } \\
\left(\left\{\varepsilon_{\mathrm{b}}\right\}_{\mathrm{p}^{\prime}}\left\{\varepsilon_{\mathrm{s}}\right\}_{\mathrm{p}}\right) & =\left(\left\{\varepsilon_{\mathrm{bt}}\right\}+\left[\mathrm{Z}_{3}\right]\left\{\varepsilon_{\mathrm{br}}\right\},\left\{\varepsilon_{\mathrm{st}}\right\}+\left[\mathrm{Z}_{6}\right]\left\{\varepsilon_{\mathrm{sr}}\right\}\right)
\end{aligned}
$$

where $\left[z_{1}\right]=\left[\left[\bar{z}_{1}\right]\left[\bar{M}_{z 1}\right]\right],\left[z_{2}\right]=\left[\left[\bar{z}_{2}\right]\left[\bar{M}_{z 2}\right]\right],\left[z_{3}\right]=\left[\left[\bar{z}_{3}\right]\left[\bar{M}_{z 3}\right]\right]$

$\left[Z_{4}\right]=\left[\bar{l}\left[\bar{M}_{Z 4}^{\prime}\right]\left[\bar{Z}_{4}\right]\left[\bar{M}_{Z 4}\right]\right],\left[Z_{5}\right]=\left[\bar{l}\left[\bar{M}_{Z 5}^{\prime}\right]\left[\bar{Z}_{5}\right]\left[\bar{M}_{Z 5}\right]\right]$

$\left[z_{6}\right]=\left[\bar{l}\left[\bar{M} z_{Z 6}^{\prime}\right]\left[\bar{Z}_{6}\right]\left[\bar{M}_{Z 6}\right]\right]$

in which $\left[\bar{Z}_{1}\right]_{k=1 \text { toN }}=\left[\bar{Z}_{2}\right]_{k=N+1}=\left[\bar{Z}_{1}\right]_{k=N+2}=\left[\begin{array}{llll}z & 0 & 0 & 0 \\ 0 & z & 0 & 0 \\ 0 & 0 & z & 0 \\ 0 & 0 & 0 & 1\end{array}\right]$

$\left[\bar{M}_{Z 1}\right]_{k=1 \text { toN }}=\left[\bar{M}_{Z 2}\right]_{k=N+1}=\left[\bar{M}_{Z 3}\right]_{k=N+2}$

$=\left[\begin{array}{cccc}(-1)^{k} \zeta_{k} & 0 & 0 & 0 \\ 0 & (-1)^{k} \zeta_{k} & 0 & 0 \\ 0 & 0 & (-1)^{k} \zeta_{k} & 0 \\ 0 & 0 & 0 & 2 z\end{array}\right]$

$[\bar{l}]=\left[\begin{array}{ll}1 & 0 \\ 0 & 1\end{array}\right],\left[\bar{Z}_{4}\right]_{k=1 \text { toN }}=\left[\bar{Z}_{5}\right]_{k=N+1}=\left[\bar{Z}_{6}\right]_{k=N+2}=\left[\begin{array}{ll}z & 0 \\ 0 & z\end{array}\right]$
$\left[\bar{M}_{Z 4}\right]_{k=1 \text { toN }}=\left[\bar{M}_{Z 5}\right]_{k=N+1}=\left[\bar{M}_{Z 6}\right]_{k=N+2}=\left[\begin{array}{cc}z^{2} & 0 \\ 0 & z^{2}\end{array}\right]$

$\left[\bar{M}_{Z 4}^{\prime}\right]_{k=1 \text { toN }}=\left[\bar{M}_{Z 4}^{\prime}\right]_{k=N+1}=\left[\bar{M}_{Z 4}^{\prime}\right]_{k=N+2}=\left[\begin{array}{cc}(-1)^{k} \frac{2}{h^{k}} & 0 \\ 0 & (-1)^{k} \frac{2}{h^{k}}\end{array}\right]$

while the vectors $\left\{\varepsilon_{\mathrm{bt}}\right\},\left\{\varepsilon_{\mathrm{br}}\right\},\left\{\varepsilon_{\mathrm{st}}\right\}$ and $\left\{\varepsilon_{\mathrm{sr}}\right\}$ appearing in Eq. (9) are given by

$$
\begin{aligned}
\begin{aligned}
\left\{\varepsilon_{\mathrm{bt}}\right\} & =\nabla_{1}\left\{\mathrm{~d}_{\mathrm{t}}\right\},\left\{\varepsilon_{\mathrm{br}}\right\}=\nabla_{2}\left\{\mathrm{~d}_{\mathrm{r}}\right\},\left\{\varepsilon_{\mathrm{st}}\right\} \\
& =\nabla_{3}\left\{\mathrm{~d}_{\mathrm{t}}\right\} \text { and }\left\{\varepsilon_{\mathrm{sr}}\right\}=\nabla_{4}\left\{\mathrm{~d}_{\mathrm{r}}\right\}
\end{aligned} \\
\text { where } \nabla_{1}=\left[\begin{array}{ccc}
\frac{\partial}{\partial x} & 0 & 0 \\
0 & \frac{\partial}{\partial y} & 0 \\
\frac{\partial}{\partial y} & \frac{\partial}{\partial x} & 0 \\
0 & 0 & 0
\end{array}\right], \nabla_{1 *}=\left[\begin{array}{ccc}
\frac{\partial}{\partial x} & 0 & 0 \\
0 & \frac{\partial}{\partial y} & 0 \\
\frac{\partial}{\partial y} & \frac{\partial}{\partial x} & 0 \\
0 & 0 & 1
\end{array}\right], \\
0^{1 *}=\left[\begin{array}{ccc}
0 & 0 & 0 \\
0 & 0 & 0 \\
0 & 0 & 0 \\
0 & 0 & 0
\end{array}\right] \nabla_{2}=\left[\begin{array}{cc}
\nabla_{1 *} & 0^{1 *} \\
0^{1 *} & \nabla_{1 *}
\end{array}\right]
\end{aligned}
$$

$I^{2}=\left[\begin{array}{lll}1 & 0 & 0 \\ 0 & 1 & 0\end{array}\right], 0^{2 *}=\left[\begin{array}{lll}0 & 0 & 0 \\ 0 & 0 & 0\end{array}\right] \nabla_{3}=\left[\begin{array}{lll}0 & 0 & \frac{\partial}{\partial x} \\ 0 & 0 & \frac{\partial}{\partial y}\end{array}\right], \nabla_{4}=\left[\begin{array}{cc}1^{2} & 0^{2 *} \\ 0^{2 *} & 1^{2} \\ \nabla_{3} & 0^{2 *} \\ 0^{2 *} & \nabla_{3}\end{array}\right]$

The stress strain constitutive relations for layer ' $\mathbf{k}$ ' of skew substrate are

$\left\{\sigma_{\mathrm{b}}^{\mathrm{k}}\right\}=\left[\begin{array}{llll}\bar{C}_{11}^{k} & \bar{C}_{12}^{k} & \bar{C}_{16}^{k} & \bar{C}_{13}^{k} \\ \bar{C}_{12}^{k} & \bar{C}_{22}^{k} & \bar{C}_{26}^{k} & \bar{C}_{23}^{k} \\ \bar{C}_{16}^{k} & \bar{C}_{26}^{k} & \bar{C}_{66}^{k} & \bar{C}_{36}^{k} \\ \bar{C}_{13}^{k} & \bar{C}_{23}^{k} & \bar{C}_{36}^{k} & \bar{C}_{33}^{k}\end{array}\right]\left\{\varepsilon_{\mathrm{b}}^{\mathrm{k}}\right\}$

and $\left\{\sigma_{\mathrm{s}}^{\mathrm{k}}\right\}=\left[\begin{array}{ll}\bar{C}_{55}^{k} & \bar{C}_{45}^{k} \\ \bar{C}_{45}^{k} & \bar{C}_{44}^{k}\end{array}\right]\left\{\varepsilon_{\mathrm{s}}^{k}\right\}(\mathbf{k}=1,2,3 \ldots \mathrm{N})$

and $\overline{\boldsymbol{C}}_{\mathrm{ij}}^{\boldsymbol{k}}(i, j=1,2,3 \ldots 6)$ are the elastic coefficients of layer ' $\boldsymbol{k}$ ' of the skew plate which are transformed based on the fiber orientation of the skew substrate. The viscoelastic constitutive relations are similar to the Eq. (11) with $(k=N+1)$ containing the complex isotropic coefficients given by

$\mathrm{G}_{\mathrm{v}}=\mathrm{G}(1+\mathrm{i})$ and $\mathrm{E}_{\mathrm{v}}=2 \mathrm{G}_{\mathrm{v}}\left(1++_{\mathrm{v}}\right)$

In which $G$ is the storage modulus $\vartheta$, is the Poisson's ratio and $\eta$ is the loss factor. The constitutive relations of the viscoelastic material layer $(k=N+1)$ are also given by Eq. (11) with complex elastic coefficients [22]. The 1-3 PZC 
constitutive relations consistent with the present analysis are given by

$$
\begin{aligned}
& \left\{\sigma_{b}^{N+2}\right\}=\left[\overline{\mathrm{C}}_{\mathrm{b}}^{N+2}\right]\left\{\varepsilon_{\mathrm{b}}^{\mathrm{N}+2}\right\}+\left[\overline{\mathrm{C}}_{\mathrm{bs}}^{\mathrm{N}+2}\right]\left\{\varepsilon_{\mathrm{s}}^{N+2}\right\}-\left\{\mathrm{e}_{\mathrm{b}}\right\} \mathrm{E}_{\mathrm{z}^{\prime}} \\
& \left\{\sigma_{\mathrm{s}}^{\mathrm{N}+2}\right\}=\left[\overline{\mathrm{C}}_{\mathrm{bs}}^{\mathrm{N}+2}\right]\left\{\varepsilon_{\mathrm{b}}^{\mathrm{N}+2}\right\}+\left[\overline{\mathrm{C}}_{\mathrm{s}}^{N+2}\right]\left\{\varepsilon_{\mathrm{s}}^{\mathrm{N}+2}\right\}-\left\{\mathrm{e}_{\mathrm{s}}\right\} \mathrm{E}_{\mathrm{z}} \\
& \text { and } \mathrm{D}_{\mathrm{z}}=\left\{\mathrm{e}_{\mathrm{b}}\right\}^{\top}\left\{\varepsilon_{\mathrm{b}}^{N+2}\right\}+\left\{\mathrm{e}_{\mathrm{s}}\right\}^{\top}\left\{\varepsilon_{\mathrm{s}}^{\mathrm{N}+2}\right\}+\bar{\varepsilon}_{33} \mathrm{E}_{\mathrm{z}} .
\end{aligned}
$$

Here, $\mathbf{E}_{\mathbf{z}}$ and $\mathbf{D}_{\mathbf{z}}$ are the $\mathbf{z}$ direction electric field and displacement vector and $\bar{\varepsilon}_{33}$ is the dielectric constant. The coupled constitutive Eqs. (13) are a result of oblique reinforcement of piezo fibers with coupled matrices $\left[\overline{\mathrm{C}}_{\mathrm{bs}}^{\mathrm{N}+2}\right]$ in Eq. (13) are given by

$$
\left[\overline{\mathrm{C}}_{\mathrm{bs}}^{\mathrm{N}+2}\right]=\left\langle\left[\begin{array}{cc}
\overline{\mathrm{C}}_{15}^{\mathrm{N}+2} & 0 \\
\overline{\mathrm{C}}_{25}^{\mathrm{N}+2} & 0 \\
0 & \overline{\mathrm{C}}_{46}^{\mathrm{N}+2} \\
\overline{\mathrm{C}}_{35}^{\mathrm{N}+2} & 0
\end{array}\right] \text { or }\left[\begin{array}{cc}
0 & \overline{\mathrm{C}}_{14}^{\mathrm{N}+2} \\
0 & \overline{\mathrm{C}}_{24}^{\mathrm{N}+2} \\
\overline{\mathrm{C}}_{56}^{\mathrm{N}+2} & 0 \\
0 & \overline{\mathrm{C}}_{34}^{\mathrm{N}+2}
\end{array}\right]\right\rangle
$$

The matrices entries are zero when $\psi=0^{0}$ of the piezofibers while the vectors $\left\{\mathbf{e}_{\mathbf{b}}\right\}$ and $\left\{\mathbf{e}_{\mathbf{s}}\right\}$ in Eq. (13) representing the transformed piezoelectric coefficients given by:

$\left\{\mathrm{e}_{\mathrm{b}}\right\}=\left[\begin{array}{llll}\overline{\mathrm{e}}_{31} & \overline{\mathrm{e}}_{32} & \overline{\mathrm{e}}_{36} & \overline{\mathrm{e}}_{33}\end{array}\right]^{\top}$ and $\left\{\mathrm{e}_{\mathrm{s}}\right\}=\left[\begin{array}{ll}\overline{\mathrm{e}}_{35} & \overline{\mathrm{e}}_{34}\end{array}\right]$

$\boldsymbol{T}_{\boldsymbol{p}}$ and $\boldsymbol{T}_{\boldsymbol{k}}$ are the potential and kinetic energies without accounting rotary inertia effects of the skew substrate/ ACLD system are [22]

$$
\begin{aligned}
T_{p}= & \frac{1}{2}\left[\sum_{k=1}^{N+2} \int_{\Omega}\left(\left\{\varepsilon_{b}^{k}\right\}^{T}\left\{\sigma_{b}^{k}\right\}+\left\{\varepsilon_{s}^{r}\right\}^{T}\left\{\sigma_{s}^{k}\right\}\right) d \Omega-\int_{\Omega} D_{z} E_{z} d \Omega\right] \\
& -\int_{A}\{d\}^{T}\{f\} d A \text { and } T_{k}=\frac{1}{2} \sum_{k=1}^{N+2} \int_{\Omega} \rho^{k}\left(\dot{u}^{2}+\dot{v}^{2}+\dot{w}^{2}\right) d \Omega
\end{aligned}
$$

Applying virtual work principle, the equations governing the dynamics of skew substrate ACLD is expressed as [23]

$$
\begin{aligned}
& \sum_{\mathrm{k}=1}^{5} \int_{\Omega}\left(\delta\left\{\mathrm{c}_{\mathrm{b}}^{\mathrm{k}}\right\}^{\top}\left\{\mathrm{\sigma}_{\mathrm{b}}^{\mathrm{k}}\right\}+\delta\left\{\mathrm{es}^{\mathrm{k}}\right\}^{\top}\left\{\sigma_{\mathrm{s}}^{\mathrm{k}}\right\}-\delta \mathrm{E}_{\mathrm{z}} \overline{\mathrm{e}}_{33} \mathrm{E}_{\mathrm{z}}-\delta\left\{\mathrm{d}_{\mathrm{t}}\right\}^{\top} \rho^{\mathrm{k}}\left\{\ddot{d}_{\mathrm{t}}\right\}\right) \mathrm{d} \Omega \\
& -\int_{A} \delta\left\{d_{t}\right\}^{\top}\{f\} d A=0
\end{aligned}
$$

in which $\boldsymbol{\rho}^{\mathbf{k}}, \mathbf{\Omega}$ are the density, volume of layer $\mathbf{k}$ while $\{\mathbf{f}\}$ is the external traction load on area $\mathbf{A}$.

\section{FE model of ACLD skew plate}

For developing the FE model, a mesh size $4 \times 4$ is selected based on the convergence and the element for the mesh is an eight noded quadrilateral element. Following Eq. (5), the elements node ' $j$ ' $\mathbf{D O F}$ are given by

$$
\left\{d_{t j}\right\}=\left[u_{0 j} v_{0 j} w_{0 j}\right]^{T} \text { and }\left\{d_{r j}\right\}=\left[\theta_{x j} \theta_{y j} \theta_{z j} \psi_{x j} \psi_{y j} \phi_{z j}\right]^{T}
$$

while the element displacement vectors $\left\{d_{[]}^{e}\right\}$ :

$$
\left\{d_{[]}\right\}=\left[N_{[1]}\right]\left\{d_{[1]}^{e}\right\} \text { in which }\left\{d_{[]}^{e}\right\}=\left[\left\{d_{[11}^{e}\right\}^{\top}\left\{d_{[12}^{e}\right\}^{\top} \cdot \cdots\left\{d_{[18}^{e}\right\}^{\top}\right]^{\top}
$$

$\left[\mathrm{N}_{[]}\right]=\left[\mathrm{N}_{[] 1} \mathrm{~N}_{[] 2} \ldots \mathrm{N}_{[] 8}\right]^{\top}$ and $\mathrm{N}_{[\mathrm{kk}}=\mathbf{n}_{\mathbf{k}} \mathrm{I}_{[]} ; \quad[] \stackrel{\text { either }}{\rightarrow}\left\{\begin{array}{c}t \text {-translational } \\ r \text {-rotational }\end{array}\right.$

$\mathbf{I}_{[]}$with [] $\rightarrow^{\text {either }} \mathbf{t}$ orr are size $\mathbf{3}$ and size $\mathbf{6}$ unit matrices and $\mathbf{n}_{\mathbf{k}}$ is the basis function of the $\mathbf{k}^{\text {th }}$ node. The strain vectors in the element consistent with Eq. (9) is as follows:

$$
\begin{aligned}
\left\{\varepsilon_{b}\right\}_{c} & =\left[B_{t b}\right]\left\{d_{t}^{e}\right\}+\left[Z_{1}\right]\left[B_{r b}\right]\left\{d_{r}^{e}\right\},\left\{\varepsilon_{s}\right\}_{c}=\left[B_{t s}\right]\left\{d_{t}^{e}\right\}+\left[Z_{4}\right]\left[B_{r s}\right]\left\{d_{r}^{e}\right\}, \\
\left\{\varepsilon_{b}\right\}_{v} & =\left[B_{t b}\right]\left\{d_{t}^{e}\right\}+\left[Z_{2}\right]\left[B_{r b}\right]\left\{d_{r}^{e}\right\},\left\{\varepsilon_{s}\right\}_{v}=\left[B_{t s}\right]\left\{d_{t}^{e}\right\}+\left[Z_{5}\right]\left[B_{r s}\right]\left\{d_{r}^{e}\right\}, \\
\text { and }\left\{\varepsilon_{b}\right\}_{p} & =\left[B_{t b}\right]\left\{d_{t}^{e}\right\}+\left[Z_{3}\right]\left[B_{r b}\right]\left\{d_{r}^{e}\right\},\left\{\varepsilon_{s}\right\}_{p}=\left[B_{t s}\right]\left\{d_{t}^{e}\right\}+\left[Z_{b}\right]\left[B_{r s}\right]\left\{d_{r}^{e}\right\} .
\end{aligned}
$$

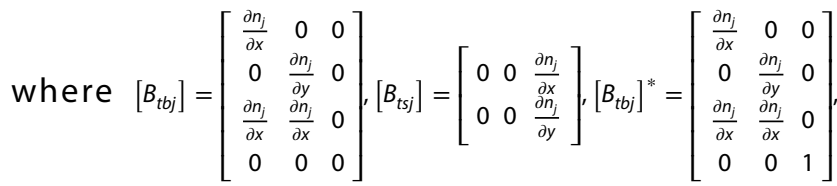
$\left[B_{r b j}\right]=\left[\begin{array}{cc}{\left[B_{t b j}\right]^{*}} & 0^{1 *} \\ 0^{1 *} & {\left[B_{t b j}\right]^{*}}\end{array}\right]$ and $\left[B_{r s j}\right]=\left[\begin{array}{cc}1^{2} & 0^{2 *} \\ 0^{2 *} & 1^{2} \\ {\left[B_{t s j}\right]} & 0^{2 *} \\ 0^{2 *} & {\left[B_{t s j}\right]}\end{array}\right]$ with elemental form being

$$
\begin{aligned}
{\left[\mathrm{B}_{\mathrm{t}()}\right] } & =\left[\begin{array}{lll}
\mathrm{B}_{\mathrm{t}(1)} & \mathrm{B}_{\mathrm{t}() 2} & \cdot \mathrm{B}_{\mathrm{t}(08}
\end{array}\right] \text { and }\left[\mathrm{B}_{\mathrm{r}(\mathrm{l}}\right] \\
& =\left[\begin{array}{lll}
\mathrm{B}_{\mathrm{r}(1)} & \mathrm{B}_{\mathrm{r}(22} \cdot \mathrm{B}_{\mathrm{r}(\mathrm{)})}
\end{array}\right] \stackrel{\text { either }}{\rightarrow}\left\{\begin{array}{c}
b \text {-bending } \\
s \text {-shear }
\end{array}\right.
\end{aligned}
$$

Putting Eqs. (11)-(15) and (21) in Eq. (17) and identifying $\mathbf{E}_{\mathbf{z}}=\mathbf{V} / \mathbf{h}_{\mathbf{p}}$ where $\mathbf{V}$ is the applied voltage to the PZC layer, the equations of motion of skew plate ACLD system as follows: 


$$
\begin{aligned}
& {\left[\mathrm{M}^{\mathrm{e}}\right]\left\{\ddot{\mathrm{d}}_{\mathrm{t}}^{\mathrm{e}}\right\}+\left[\left[\mathrm{K}_{\mathrm{tt}}^{\mathrm{e}}\right]+\left[\mathrm{K}_{\mathrm{tr}}^{\mathrm{e}}\right]^{\mathrm{T}}\right]\left\{\mathrm{d}_{\mathrm{t}}^{\mathrm{e}}\right\}+\left[\left[\mathrm{K}_{\mathrm{tr}}^{\mathrm{e}}\right]\right.} \\
& \left.+\left[\mathrm{K}_{\mathrm{rr}}^{\mathrm{e}}\right]\right]\left\{\mathrm{d}_{\mathrm{r}}^{\mathrm{e}}\right\}+\left[\left\{\mathrm{F}_{\mathrm{tp}}^{\mathrm{e}}\right\}+\left\{\mathrm{F}_{\mathrm{rp}}^{\mathrm{e}}\right\}\right] V=\left\{\mathrm{F}^{\mathrm{e}}\right\}
\end{aligned}
$$

Before deriving the global equations of the skew substrate ACLD plate, the skewed nature of the plate makes the assembly process difficult since the edges of the plate are not parallel to the reference coordinates. This makes the processes of defining the end condition in generalized displacements difficult and hence for successful implementation of the same, the skew edge transformed displacements are [23]:

$$
\left\{d_{t}\right\}=\left[T_{t}\right]\left\{d_{t}^{\prime}\right\},\left\{d_{r}\right\}=\left[T_{r}\right]\left\{d_{r}^{\prime}\right\}
$$

where $\left\{d_{t}^{\prime}\right\}$ and $\left\{d_{r}^{\prime}\right\}$ are transformed vectors in the $\left(\mathbf{x}^{\prime} \mathbf{y}^{\prime}\right.$ $\left.z^{\prime}\right)$ system given by:

$$
\left\{d_{t}^{\prime}\right\}=\left[u_{0}^{\prime} v_{0}^{\prime} w_{0}^{\prime}\right]^{\top} \text { and }\left\{d_{r}^{\prime}\right\}=\left[\theta_{x}^{\prime} \theta_{y}^{\prime} \theta_{z}^{\prime} \psi_{x}^{\prime} \psi_{y}^{\prime} \psi_{z}^{\prime}\right]^{\top}
$$

while the transformations matrices

$$
\left[T_{t}\right]=\left[\begin{array}{ccc}
c & s & 0 \\
-s & c & 0 \\
0 & 0 & 1
\end{array}\right] \text { and }\left[T_{r}\right]=\left[\begin{array}{cc}
{\left[T_{t}\right]} & O_{3} \\
O_{3} & {\left[T_{t}\right]}
\end{array}\right]
$$

in which $c=\cos \alpha, s=\sin \alpha$, and $O_{3}$ is $3 \times 3$ null matrix. The entries to the matrices of elements with nodes along the skew edge are given by

$\left[\overline{\mathrm{K}}_{\mathrm{tt}}^{\mathrm{e}}\right]=\left[\mathrm{T}_{1}\right]^{\mathrm{T}}\left[\mathrm{K}_{\mathrm{tt}}^{\mathrm{e}}\right]\left[\mathrm{T}_{1}\right],\left[\overline{\mathrm{K}}_{\mathrm{tr}}^{\mathrm{e}}\right]=\left[\mathrm{T}_{1}\right]^{\mathrm{T}}\left[\mathrm{K}_{\mathrm{tr}}^{\mathrm{e}}\right]\left[\mathrm{T}_{2}\right]$,

$\left[\overline{\mathrm{K}}_{\mathrm{rr}}^{\mathrm{e}}\right]=\left[\mathrm{T}_{2}\right]^{\top}\left[\mathrm{K}_{\mathrm{rr}}^{\mathrm{e}}\right]\left[\mathrm{T}_{2}\right]$ and $\left[\overline{\mathrm{M}}^{\mathrm{e}}\right]=\left[\mathrm{T}_{1}\right]^{\top}\left[\mathrm{M}^{\mathrm{e}}\right]\left[\mathrm{T}_{1}\right]$

where the mass and stiffness matrices $\left[\mathbf{M}^{\mathbf{e}}\right],\left[\mathbf{K}_{\mathrm{tt}}^{\mathbf{e}}\right],\left[\mathbf{K}_{\mathrm{tr}}^{\mathbf{e}}\right]$ and $\left[\mathbf{K}_{\mathbf{r r}}^{\mathbf{e}}\right]$, and the load, coupled elasticelectro vectors $\left\{F^{e}\right\},\left\{\begin{array}{l}F_{t p}^{e} \\ e\end{array},\left\{F_{r p}^{e}\right\}\right.$; in the element form seen in Eq. (25) while the transformation matrices $\left[T_{1}\right]$ and $\left[T_{2}\right]$ in Eq. (27) are as follows:

Mass Matrix : $\left[M^{e}\right]=\int_{A} \bar{m}\left[N_{t}\right]^{\top}\left[N_{t}\right] d A$ mass parameter $(\bar{m})$

$$
=\sum_{k=1}^{N+2} \rho^{k}\left(h_{k+1}-h_{k}\right)
$$

Stiff ness matrices : $\left[\mathrm{K}_{\mathrm{tt}}^{\mathrm{e}}\right]=\left[\mathrm{K}_{\mathrm{tb}}^{\mathrm{e}}\right]+\left[\mathrm{K}_{\mathrm{ts}}^{\mathrm{e}}\right]+\left[\mathrm{K}_{\mathrm{tbs}}^{\mathrm{e}}\right]_{\mathrm{pb}}+\left[\mathrm{K}_{\mathrm{tbs}}^{\mathrm{e}}\right]_{\mathrm{ps}}$

$$
\begin{aligned}
& {\left[\mathrm{K}_{\mathrm{tr}}^{\mathrm{e}}\right]=\left[\mathrm{K}_{\mathrm{trb}}^{\mathrm{e}}\right]+\left[\mathrm{K}_{\mathrm{trs}}^{\mathrm{e}}\right]+\frac{1}{2}\left(\left[\mathrm{~K}_{\mathrm{trbs}}^{\mathrm{e}}\right]_{\mathrm{pb}}+\left[\mathrm{K}_{\mathrm{rtbs}}^{\mathrm{e}}\right]_{\mathrm{pb}}^{\top}+\left[\mathrm{K}_{\mathrm{trbs}}^{\mathrm{e}}\right]_{\mathrm{ps}}+\left[\mathrm{K}_{\mathrm{rtbs}}^{\mathrm{e}}\right]_{\mathrm{ps}}^{\top}\right)} \\
& {\left[\mathrm{K}_{\mathrm{rt}}^{\mathrm{e}}\right]=\left[\mathrm{K}_{\mathrm{tr}}^{\mathrm{e}}\right]^{\top},\left[\mathrm{K}_{\mathrm{rr}}^{\mathrm{e}}\right]=\left[\mathrm{K}_{\mathrm{rrb}}^{\mathrm{e}}\right]+\left[\mathrm{K}_{\mathrm{rrs}}^{\mathrm{e}}\right]+\left[\mathrm{K}_{\mathrm{rrbs}}^{\mathrm{e}}\right]_{\mathrm{pb}}+\left[\mathrm{K}_{\mathrm{rrbs}}^{\mathrm{e}}\right]_{\mathrm{ps}}}
\end{aligned}
$$

Electro-elastic coupling vectors : $\left\{\mathrm{F}_{\mathrm{tp}}^{\mathrm{e}}\right\}$

$$
\begin{aligned}
& =\left\{\mathrm{F}_{\mathrm{rb}}^{\mathrm{e}}\right\}_{\mathrm{p}}+\left\{\mathrm{F}_{\mathrm{rs}}^{\mathrm{e}}\right\}_{\mathrm{p}} \\
& \text { and Load vector : } \\
& =\int_{\mathrm{A}}\left[\mathrm{N}_{\mathrm{t}}\right]^{\top}\{\mathrm{f}\} \mathrm{dA}
\end{aligned}
$$$$
=\left\{\mathrm{F}_{\mathrm{tb}}^{\mathrm{e}}\right\}_{\mathrm{p}}+\left\{\mathrm{F}_{\mathrm{ts}}^{\mathrm{e}}\right\}_{\mathrm{p}^{\prime}}\left\{\mathrm{F}_{\mathrm{rp}}^{\mathrm{e}}\right\}
$$$$
\text { and Load vector : }\left\{\mathrm{F}^{\mathrm{e}}\right\}
$$

in which $\left[K_{\mathrm{t}()}^{e}\right]=\int_{A}\left[B_{\mathrm{t}()}\right]^{T}\left(\left[D_{\mathrm{t}()}\right]+\left[D_{\mathrm{t}()}\right]_{V}+\left[D_{\mathrm{t}()}\right]_{p}\right)\left[B_{\mathrm{t}()}\right] \mathrm{dA}$,

$\left[K_{\operatorname{tr}()}^{e}\right]=\int_{A}\left[B_{\operatorname{tr}()}\right]^{T}\left(\left[D_{\operatorname{tr}()}\right]+\left[D_{\operatorname{tr}()}\right]_{V}+\left[D_{\operatorname{tr}()}\right]_{p}\right)\left[B_{\operatorname{tr}()}\right] \mathrm{dA}$

$\left[K_{\mathrm{rr}()}^{e}\right]=\int_{A}\left[B_{\mathrm{rr}()}\right]^{T}\left(\left[D_{\mathrm{rr}()}\right]+\left[D_{\mathrm{rr}()}\right]_{V}+\left[D_{\mathrm{rr}()}\right]_{p}\right)\left[B_{\mathrm{rr}()}\right] \mathrm{dA}$

()$^{\text {either }} \rightarrow\left\{\begin{array}{c}b \text {-bending } \\ s \text {-shear }\end{array}, \mathrm{dA}=\mathrm{dxdy}\right.$

$\left[K_{\mathrm{tbs}}^{e}\right]_{p b}=\int_{A}\left[B_{\mathrm{tb}}\right]^{T}\left[D_{\mathrm{tbs}}\right]_{p}\left[B_{\mathrm{ts}}\right] \mathrm{dA}$,

$\left[K_{\mathrm{tbs}}^{e}\right]_{p s}=\int_{A}\left[B_{\mathrm{ts}}\right]^{T}\left[D_{\mathrm{tbs}}\right]_{p}\left[B_{\mathrm{tb}}\right] \mathrm{dA}$

$\left[K_{\mathrm{trbs}}^{e}\right]_{p b}=\int_{A}\left[B_{\mathrm{tb}}\right]^{T}\left[D_{\mathrm{trbs}}\right]_{p}\left[B_{\mathrm{rs}}\right] \mathrm{dA}$,

$\left[K_{\mathrm{rtbs}}^{e}\right]_{p b}=\int_{A}\left[B_{\mathrm{rb}}\right]^{T}\left[D_{\mathrm{rtbs}}\right]_{p}\left[B_{\mathrm{ts}}\right] \mathrm{dA}$

$\left[K_{\mathrm{trbs}}^{e}\right]_{p s}=\int_{A}\left[B_{\mathrm{ts}}\right]^{T}\left[D_{\mathrm{rtbs}}\right]_{p}\left[B_{\mathrm{rb}}\right] \mathrm{dA}$,

$\left[K_{\mathrm{rtbs}}^{e}\right]_{p s}=\int_{A}\left[B_{\mathrm{rs}}\right]^{T}\left[\mathrm{Dr}_{\mathrm{trbs}}\right]_{p}\left[B_{\mathrm{tb}}\right] \mathrm{dA}$ 


$$
\begin{aligned}
& {\left[K_{\mathrm{rrbs}}^{e}\right]_{p b}=\int_{A}\left[B_{\mathrm{rb}}\right]^{T}\left[D_{\mathrm{rrbs}}\right]_{p}\left[B_{\mathrm{rs}}\right] \mathrm{dA}} \\
& {\left[K_{\mathrm{rrbs}}^{e}\right]_{p s}=\int_{A}\left[B_{\mathrm{rs}}\right]^{T}\left[D_{\mathrm{rrbs}}\right]_{p}\left[B_{\mathrm{rb}}\right] \mathrm{dA}} \\
& \left\{F_{t b}^{e}\right\}_{p}=\int_{A}\left[B_{\mathrm{tb}}\right]^{T}\left\{D_{\mathrm{tb}}\right\}_{p} \mathrm{dA},\left\{F_{t s}^{e}\right\}_{p}=\int_{A}\left[B_{\mathrm{ts}}\right]^{T}\left\{D_{\mathrm{ts}}\right\}_{p} \mathrm{dA} \\
& \text { and }\left\{F_{r s}^{e}\right\}_{p}=\int_{A}\left[B_{\mathrm{rs}}\right]^{T}\left\{D_{\mathrm{rs}}\right\}_{p} \mathrm{dA} \\
& \text { where }\left[D_{t b}\right]=\sum_{k=1}^{N} \int_{h_{k}}^{h_{k+1}}\left[\bar{C}_{b}^{k}\right] d z,\left[D_{t s}\right]=\sum_{k=1}^{N} \int_{h_{k}}^{h_{k+1}}\left[\overline{\bar{C}}_{s}^{k}\right] d z \\
& {\left[D_{t r b}\right]=\sum_{k=1}^{N} \int_{h_{k}}^{h_{k+1}}\left[\bar{C}_{b}^{k}\right]\left[Z_{1}\right] d z,\left[D_{t r s}\right]=\sum_{k=1}^{N} \int_{h_{k}}^{h_{k+1}}\left[\bar{C}_{s}^{k}\right]\left[Z_{4}\right] d z} \\
& {\left[D_{r r b}\right]=\sum_{k=1}^{N} \int_{h_{k}}^{h_{k+1}}\left[Z_{1}\right]^{T}\left[\bar{C}_{b}^{k}\right]\left[Z_{1}\right] d z_{,}\left[D_{r r s}\right]=\sum_{k=1}^{N} \int_{h_{k}}^{h_{k+1}}\left[Z_{4}\right]^{T}\left[\bar{C}_{s}^{k}\right]\left[Z_{4}\right] d z} \\
& {\left[D_{t b}\right]_{v}=h_{v}\left[\bar{C}_{b}^{N+1}\right],\left[D_{t r b}\right]_{v}=\int_{h_{N+1}}^{h_{v}}\left[\bar{C}_{b}^{N+1}\right]\left[Z_{2}\right] \mathrm{dz},\left[D_{r r b}\right]_{v}} \\
& \begin{aligned}
{\left[D_{t s}\right]_{p} } & =h_{p}\left[\bar{C}_{s}^{N+2}\right],\left[D_{t r s}\right]_{p}=\int_{h_{v}}^{h_{p}}\left[\bar{C}_{s}^{N+2}\right]\left[Z_{6}\right] \mathrm{dz},\left[D_{r r s}\right]_{p} \\
& =\int_{h_{v}}^{h_{p}}\left[Z_{6}\right]^{T}\left[\bar{C}_{b}^{N+2}\right]\left[Z_{6}\right] \mathrm{d} z
\end{aligned} \\
& {\left[D_{t b s}\right]_{p}=\int_{h_{v}}^{h_{p}}\left[\bar{C}_{b s}^{N+2}\right] \mathrm{dz},\left[D_{t r b s}\right]_{p}=\int_{h_{v}}^{h_{p}}\left[\bar{C}_{b s}^{N+2}\right]\left[Z_{6}\right] \mathrm{dz},\left[D_{r t b s}\right]_{p}} \\
& =\int_{h_{v}}^{h_{p}}\left[Z_{3}\right]^{T}\left[\bar{C}_{b s}^{N+2}\right] d z \\
& {\left[D_{r r b s}\right]_{p}=\int_{h_{v}}^{h_{p}}\left[Z_{3}\right]^{T}\left[\bar{C}_{b s}^{N+3}\right]\left[Z_{6}\right] \mathrm{dz},\left\{D_{t b}\right\}_{p}=\int_{h_{v}}^{h_{p}}-\frac{\left\{\bar{e}_{b}\right\}}{h_{p}} \mathrm{dz},} \\
& \left\{D_{r b}\right\}_{p}=\int_{h_{v}}^{h_{p}}-\left[Z_{3}\right]^{T} \frac{\left\{\bar{e}_{b}\right\}}{h_{p}} \mathrm{dz},\left\{D_{t s}\right\}_{p}=\int_{h_{v}}^{h_{p}}-\frac{\left\{\bar{e}_{s}\right\}}{h_{p}} \mathrm{dz}, \\
& \left\{D_{r s}\right\}_{p}=\int_{h_{v}}^{h_{p}}-\left[Z_{6}\right]^{T} \frac{\left\{\bar{e}_{s}\right\}}{h_{p}} \mathrm{dz} .
\end{aligned}
$$$$
=\int_{h_{N+1}}^{h_{v}}\left[Z_{2}\right]^{T}\left[\bar{C}_{b}^{N+1}\right]\left[Z_{2}\right] d z
$$$$
\left[D_{t s}\right]_{V}=h_{v}\left[\bar{C}_{s}^{N+1}\right],\left[D_{t r s}\right]_{V}=\int_{h_{N+1}}^{h_{V}}\left[\bar{C}_{s}^{N+1}\right]\left[Z_{5}\right] \mathrm{dz},\left[D_{r r s}\right]_{V}
$$$$
=\int_{h_{N+1}}^{h_{v}}\left[Z_{5}\right]^{T}\left[\bar{C}_{b}^{N+1}\right]\left[Z_{5}\right] d z
$$$$
\left[D_{t b}\right]_{p}=h_{p}\left[\bar{C}_{b}^{N+2}\right],\left[D_{t r b}\right]_{p}=\int_{h_{v}}^{h_{p}}\left[\bar{C}_{b}^{N+2}\right]\left[Z_{3}\right] \mathrm{dz},\left[D_{r r b}\right]_{p}
$$$$
=\int_{h_{v}}^{h_{p}}\left[Z_{3}\right]^{T}\left[\bar{C}_{b}^{N+2}\right]\left[Z_{3}\right] d z
$$

The Transformation matrices $\left[T_{1}\right],\left[T_{2}\right]$ appearing in Eq. (27) are given by

$$
\begin{aligned}
& {\left[\mathrm{T}_{1}\right]=\left[\begin{array}{llllll}
{\left[\mathrm{T}_{\mathrm{t}}\right]} & \mathrm{O}_{3} & \mathrm{O}_{3} & \mathrm{O}_{3} & \mathrm{O}_{3} & \mathrm{O}_{3} \\
\mathrm{O}_{3} & {\left[\mathrm{~T}_{\mathrm{t}}\right]} & \mathrm{O}_{3} & \mathrm{O}_{3} & \mathrm{O}_{3} & \mathrm{O}_{3} \\
\mathrm{O}_{3} & \mathrm{O}_{3} & {\left[\mathrm{~T}_{\mathrm{t}}\right.} & \mathrm{O}_{3} & \mathrm{O}_{3} & \mathrm{O}_{3} \\
\mathrm{O}_{3} & \mathrm{O}_{3} & \mathrm{O}_{3} & {\left[\mathrm{~T}_{\mathrm{t}}\right]} & \mathrm{O}_{3} & \mathrm{O}_{3} \\
\mathrm{O}_{3} & \mathrm{O}_{3} & \mathrm{O}_{3} & \mathrm{O}_{3} & {\left[\mathrm{~T}_{\mathrm{t}}\right]} & \mathrm{O}_{3} \\
\mathrm{O}_{3} & \mathrm{O}_{3} & \mathrm{O}_{3} & \mathrm{O}_{3} & \mathrm{O}_{3} & {\left[\mathrm{~T}_{\mathrm{t}}\right]}
\end{array}\right]} \\
& {\left[\mathrm{T}_{2}\right]=\left[\begin{array}{llllll}
{\left[\mathrm{T}_{\mathrm{r}}\right]} & \mathrm{O}_{6} & \mathrm{O}_{6} & \mathrm{O}_{6} & \mathrm{O}_{6} & \mathrm{O}_{6} \\
\mathrm{O}_{6} & {\left[\mathrm{~T}_{\mathrm{r}}\right]} & \mathrm{O}_{6} & \mathrm{O}_{6} & \mathrm{O}_{6} & \mathrm{O}_{6} \\
\mathrm{O}_{6} & \mathrm{O}_{6} & {\left[\mathrm{~T}_{\mathrm{r}}\right]} & \mathrm{O}_{6} & \mathrm{O}_{6} & \mathrm{O}_{6} \\
\mathrm{O}_{6} & \mathrm{O}_{6} & \mathrm{O}_{6} & {\left[\mathrm{~T}_{\mathrm{r}}\right]} & \mathrm{O}_{6} & \mathrm{O}_{6} \\
\mathrm{O}_{6} & \mathrm{O}_{6} & \mathrm{O}_{6} & \mathrm{O}_{6} & {\left[\mathrm{~T}_{\mathrm{r}}\right]} & \mathrm{O}_{6} \\
\mathrm{O}_{6} & \mathrm{O}_{6} & \mathrm{O}_{6} & \mathrm{O}_{6} & \mathrm{O}_{6} & {\left[\mathrm{~T}_{\mathrm{r}}\right.}
\end{array}\right]}
\end{aligned}
$$

in which $\mathrm{O}_{3}$ and $\mathrm{O}_{6}$ are $(3 \times 3)$ and $(6 \times 6)$ null matrices, respectively. Assembling the element equations in the corresponding manner results in global equations of motion given by 


$$
\begin{aligned}
& {[\mathrm{M}]\{\ddot{\mathrm{X}}\}+\left(\left[\mathrm{K}_{\mathrm{tt}}\right]+\left[\mathrm{K}_{\mathrm{tr}}\right]^{\top}\right)\{\mathrm{X}\}+\left(\left[\mathrm{K}_{\mathrm{tr}}\right]+\left[\mathrm{K}_{\mathrm{rr}}\right]\right)\left\{\mathrm{X}_{\mathrm{r}}\right\}} \\
& +\sum_{\mathrm{p}=1}^{\mathrm{q}}\left(\left\{\mathrm{F}_{\mathrm{tp}}^{\mathrm{p}}\right\}+\left\{\mathrm{F}_{\mathrm{rp}}^{\mathrm{p}}\right\}\right) \mathrm{V}^{\mathrm{p}}=\{\mathrm{F}\}
\end{aligned}
$$

where the matrices in Eq. (28) are similar to those in Eq. (23) in the global form, $\{\mathbf{X}\}$ and $\left\{\mathbf{X}_{\mathbf{r}}\right\}$ are the DOF representing translational and rotational displacements, $\mathbf{V}^{\mathbf{p}}$-voltage applied and $\mathbf{P}$-patch number. The entries to the stiffness matrices in Eq. (28) are complex due to the viscoelastic material, the energy loss in the system is ascribed to the imaginary parts. The Eq. (28) also amounts for uncontrolled responses of the ACLD skew plate taking $\mathbf{V}^{\mathbf{p}}=0$ (for all $\left.p\right)$.

\section{Closed-loop model}

For active mode, the ACLD patches are activated by supply of control voltage at a particular point as a function of transverse velocity of the same. The supply voltage in terms of control gain $\mathbf{K}_{\mathbf{d}}^{\mathbf{p}}$ is expressed as:

$\mathrm{V}^{\mathrm{p}}=-\mathrm{K}_{\mathrm{d}}^{\mathrm{p}} \dot{\mathrm{w}}=-\mathrm{K}_{\mathrm{d}}^{\mathrm{p}}\left(\left[\mathrm{U}_{\mathrm{t}}^{\mathrm{p}}\right]\{\dot{\mathrm{X}}\}-\left(\frac{\mathrm{h}}{2}\right)\left[\mathrm{U}_{\mathrm{r}}^{\mathrm{p}}\right]\left\{\dot{\mathrm{X}}_{\mathrm{r}}\right\}\right)$

In which $\left[\mathbf{U}_{\mathbf{t}}^{\mathbf{p}}\right]$ and $\left[\mathbf{U}_{\mathbf{r}}^{\mathbf{p}}\right]$ are the transverse unit vectors of voltage supply. The equations in the final form adopting the feedback system are found by substitution of the Eqs. (29) in (28) as:

$$
\begin{aligned}
& {[\mathrm{M}]\{\ddot{\mathrm{X}}\}+\left[\left[\mathrm{K}_{\mathrm{tt}}\right]+\left[\mathrm{K}_{\mathrm{rt}}\right]\right]\{\mathrm{X}\}+\left[\left[\mathrm{K}_{\mathrm{tr}}\right]+\left[\mathrm{K}_{\mathrm{rr}}\right]\right]\left\{\mathrm{K}_{\mathrm{r}}\right\}} \\
& \quad+2\left\{\sum_{p=1}^{m}\left[K_{d}^{p}\left\{F_{t p}^{p}\right\}\left[U_{t}^{p}\right]\{\dot{X}\}+K_{d}^{p}\left(\frac{h}{2}\right)\left\{F_{t p}^{p}\right\}\left[U_{r}^{p}\right]\left\{\dot{X}_{r}\right\}\right]\right\}=\{\mathrm{F}\}
\end{aligned}
$$

\section{Results and discussion}

The model derived in the earlier section are utilized to present the findings of the study. The dimensions of the skew substrate ACLD system are shown in Table 1 while the properties of the constituent materials of the ACLD skew substrate are listed in Table 2. The composition of the smart constraining layer is $60 \%$ fiber volume fraction PZT$5 \mathrm{H} /$ spur composite [19]. To carry out the analysis, different end conditions of the skew plates are considered and the same are listed in Table 3. To proceed with the analysis, the first step is to validate the developed FE model with incorporated MZZF, the same is used for evaluating the transverse displacements and fundamental frequency parameters of similar plates available in the literature. Tables 4 and 5 list the evaluations of the same and corresponding values are compared with the works of earlier researchers $[4,30]$. The results shown in the Tables 4 and 5 are found to be in good agreement with the benchmark works of Garg et al. [4] and Reddy [30]. Also, these results not only clearly establish the present model but also proves to be versatile for predicting the behavior of thicker plates. The next step in the analysis is to use the present model to evaluate the frequency response functions (FRFs)skew substrate plate ACLD system and assess the damping behavior of the same. For such a study a force $1 \mathbf{N}$ magnitude of time harmonic nature is applied at the point $(\mathbf{a} / \mathbf{2 +} \mathbf{( b / 4 )} \sin \mathbf{a}$, $(\mathbf{b} / \mathbf{4}) \cos \mathbf{a}, \mathbf{h} / \mathbf{2}$ ) on the top surface of the skew substrate while the control voltages for activating the patches are supplied to the points $(a / 2+(b / 4) \sin a,(b / 4) \cos a, h / 2)$ and $(\mathbf{a} / \mathbf{2}+(\mathbf{b} / \mathbf{4}) \sin \mathbf{a},(\mathbf{3} \mathbf{b} / \mathbf{4}) \cos \mathbf{a}, \mathbf{h} / \mathbf{2})$. Except stated, the aspect ratio $(\mathbf{a} / \mathbf{h})$ is 100 , the piezo fibers of smart layer are vertically aligned $\left(\boldsymbol{\Psi}=0^{\circ}\right)$ and the skew angle $\left(\boldsymbol{a}=30^{\circ}\right)$ are considered for further analysis. The controlled FRFs of SS1 skew plates composed of symmetric cross-ply $\left(0^{\circ} / 90^{\circ} / 0^{\circ}\right)$ substrate laminates for different skew angles are displayed in Figs. 4, 5 and 6, while the equivalent required voltages are illustrated in Figs. 7, 8 and 9. It can be seen from these figures that the present model incorporating the Zig-Zag theory is found to be very effective in damping the responses of the skew plates. Also, the results shown above clearly provide an insight into modelling the smart damping responses of the structures which has not been attempted earlier and can be treated as benchmark results for future research in the similar area. Similar results not shown here for brevity sake have been obtained for antisymmetric cross ply $\left(0^{\circ} / 90^{\circ} / 0^{\circ} / 90^{\circ}\right)$ and antisymmetric angle-ply $\left(-45^{\circ} / 45^{\circ} /-45^{\circ} / 45^{\circ}\right)$ skew substrates. The contribution of the transverse actuation for improving the damping behavior of ACLD patch for controlling the vibrations of SS 1 symmetric cross-ply $\left(0^{\circ} / 90^{\circ} / 0^{\circ}\right)$ skew substrate with $\mathbf{a}=30^{\circ}$ is shown in Fig. 10. The responses show that the effect of considering e 33 and $\mathbf{e} 31$ contributes significantly in damping these responses in comparison to the in-plane actuation. Also, the effect of piezoelectric coefficient e33 is found to be significant over e31 for the same which justifies consideration of transverse normal strain for better performance evaluation. In order to investigate

Table 1 Dimensions of the skew substrate ACLD system

\begin{tabular}{ll}
\hline Skew substrate ACLD system & Notation with dimensions \\
\hline Thickness of skew substrate & $\mathbf{h}=0.003 \mathrm{~m}$ \\
Aspect ratio of substrate & $\mathbf{a} / \mathbf{h}=100$ \\
Length/width of the plate & $\mathbf{a}=0.3 \mathrm{~m}$ \\
Thickness of the ACLD patch & $\mathbf{h}_{\mathbf{p}}+\mathbf{h}_{\mathbf{v}}$ \\
& $\mathbf{h}_{\mathbf{p}}(1-3$ PZC layer $)-200 \mu \mathrm{m}$ \\
& $\mathbf{h}_{\mathbf{v}}($ Viscoelastic layer $)-50 \mu \mathrm{m}$ \\
\end{tabular}


Table 2 Material properties of the components of the ACLD skew plate

Table 3 Types of Support end conditions of the plate

\begin{tabular}{lll}
\hline Component & Layer number & Material properties \\
\hline Skew substrate [30] & $k=(1,2,3 \ldots . N)$ & $E_{1}=172 \mathrm{GPa}, \mathrm{E}_{1} / \mathrm{E}_{2}=25$, \\
& $\mathrm{G}_{12}=\mathrm{G}_{13}=0.5 \mathrm{E}_{2}, \mathrm{G}_{23}=0.5 \mathrm{E}_{2}$, \\
& $\mathrm{v}_{13}=\mathrm{v}_{13}=\mathrm{v}_{13}=0.25$ \\
& Shear Modulus $\mathbf{G}=20(1+i) \mathrm{MN} / \mathrm{m}^{2}$, \\
& $\begin{array}{l}\text { Poisson ratio } \mathbf{v}=0.49 \\
\text { Viscoelastic layer [22] }\end{array}$ & and $\boldsymbol{\rho}=1140 \mathrm{~kg} / \mathrm{m}^{3}$ \\
& $C_{11}=9.29 \mathrm{GPa}, C_{12}=6.18 \mathrm{GPa}$, \\
& $C_{13}=6.05 \mathrm{GPa}, C_{33}=35.44 \mathrm{GPa}$, \\
& $C_{23}=C_{13}, C_{55}=C_{44}, C_{44}=1.58 \mathrm{GPa}$, \\
& $C_{33}=1.54 \mathrm{GPa}, e_{31}=-0.1902 \mathrm{C} / \mathrm{m}^{2}$, \\
& $e_{32}=e_{31}, e_{24}=0.004 \mathrm{C} / \mathrm{m}^{2}$, \\
& $e_{33}=18.4107 \mathrm{C} / \mathrm{m}^{2}$ and $e_{15}=e_{24}$, \\
\hline
\end{tabular}

\begin{tabular}{|c|c|c|}
\hline End condition & $x=0$ and $x=a$ & $\mathrm{y}=0$ and $\mathrm{y}=b$ \\
\hline Simply-supported (SS1) & $v_{0}=w_{0}=\theta_{y}=\psi_{y}=\theta_{z}=\psi_{z}=0$ & $u_{0}=w_{0}=\theta_{x}=\psi_{x}=\theta_{z}=\psi_{z}=0$ \\
\hline Simply-supported(SS2) & $u_{0}=w_{0}=\theta_{y}=\psi_{y}=\theta_{z}=\psi_{z}=0$ & $v_{0}=w_{0}=\theta_{x}=\psi_{x}=\theta_{z}=\psi_{z}=0$ \\
\hline Clamped-clamped(CC) & $\begin{array}{l}u_{0}=v_{0}=w_{0}=\theta_{x}=\psi_{x} \\
\quad=\theta_{y}=\psi_{y}=\theta_{z}=\psi_{z}=0\end{array}$ & $\begin{array}{l}u_{0}=v_{0}=w_{0}=\theta_{x}=\psi_{x} \\
\quad=\theta_{y}=\psi_{y}=\theta_{z}=\psi_{z}=0\end{array}$ \\
\hline
\end{tabular}

\begin{tabular}{|c|c|c|c|c|c|c|c|}
\hline \multirow[t]{2}{*}{$\begin{array}{l}\text { Skew } \\
\text { angle }(\alpha)\end{array}$} & \multirow[t]{2}{*}{ Source } & \multicolumn{2}{|c|}{$\begin{array}{l}\text { Symmetric cross-ply } \\
90^{\circ} / 0^{\circ} / 90^{\circ} / 0^{\circ} / 90^{\circ}\end{array}$} & \multicolumn{2}{|c|}{$\begin{array}{l}\text { Antisymmetric cross- } \\
\text { ply } \\
\left(0^{0} / 90^{\circ} / 0^{\circ} / 90^{\circ}\right)\end{array}$} & \multicolumn{2}{|c|}{$\begin{array}{l}\text { Antisymmetric } \\
\text { angle-ply } \\
45^{0} /-45^{0} / 45^{\circ} /-45^{0}\end{array}$} \\
\hline & & Mode-1 & Mode-2 & Mode-1 & Mode-2 & Mode-1 & Mode-2 \\
\hline \multirow[t]{4}{*}{0} & Ref. [4] & 1.5699 & 3.0372 & 1.5119 & 2.4656 & 1.9171 & 3.4869 \\
\hline & & 1.5703 & 2.8917 & 1.4829 & 2.4656 & 1.7974 & 3.3351 \\
\hline & Ref. [30] & 1.5635 & 2.4383 & 1.5076 & 2.4380 & 1.8493 & 3.3359 \\
\hline & Present MZZF & 1.5779 & 2.4392 & 1.5197 & 2.4392 & 1.8779 & 3.4220 \\
\hline \multirow[t]{4}{*}{15} & Ref. [4] & 1.6874 & 3.1413 & 1.6081 & 2.5364 & 1.9366 & 3.4206 \\
\hline & & 1.6877 & 3.0458 & 1.5741 & 2.5351 & 1.8313 & 3.2490 \\
\hline & Ref. [30] & 1.6571 & 2.9840 & 1.5796 & 2.5775 & 1.8675 & 3.2075 \\
\hline & Present MZZF & 1.6751 & 3.0223 & 1.5945 & 2.5771 & 1.8970 & 3.2857 \\
\hline \multirow[t]{4}{*}{30} & Ref. [4] & 2.0884 & 3.5147 & 1.9439 & 2.9389 & 2.1196 & 3.6146 \\
\hline & & 2.0840 & 3.4023 & 1.8871 & 2.9372 & 2.0270 & 3.4431 \\
\hline & Ref. [30] & 1.9596 & 3.1690 & 1.8226 & 2.9585 & 1.9894 & 3.2365 \\
\hline & Present MZZF & 1.9900 & 3.2404 & 1.8473 & 2.9576 & 2.0238 & 3.3156 \\
\hline \multirow[t]{4}{*}{45} & Ref. [4] & 2.8932 & 4.2852 & 2.6752 & 3.3131 & 2.6752 & 3.3131 \\
\hline & & 2.8925 & 4.1906 & 2.5609 & 3.3126 & 2.5609 & 3.3125 \\
\hline & Ref. [30] & 2.4811 & 4.4875 & 2.2996 & 3.4773 & 2.3194 & 3.4870 \\
\hline & Present MZZF & 2.5349 & 4.6255 & 2.3464 & 3.5708 & 2.3676 & 3.5809 \\
\hline
\end{tabular}

$\bar{\omega}=\left(\omega b^{2} / \pi^{2} h\right) \sqrt{\rho / E_{2}}, \omega$ is the circular natural frequency

Table 4 Fundamental frequency $(\overline{\boldsymbol{\omega}})$ of SS1 Skew substrate laminates with ACLD of negligible thickness $(\mathbf{a}=\mathbf{b})$ responses has been found when the value $\boldsymbol{\Psi}$ is $0^{0}$ for SS1 end condition while the same is found maximum when $\Psi$ is $30^{\circ}$ for CC end conditions, respectively. Similar results not shown for brevity sake are obtained for other substrate skew plates with coplanar yz-plane piezo fibers. plates with coplanar $\mathbf{x z}$-plane piezofibers for different ' $\boldsymbol{\Psi}^{\prime}$ (i.e., $\boldsymbol{\Psi}=0^{0}, 15^{0}, 30^{\circ}, 45^{\circ}$ ), respectively. It may be observed that maximum attenuation of the patch for controlling the control authority of the patch, the ' $\boldsymbol{\psi}^{\prime}$ of oblique coplanar xz- and yz-plane fibers of 1-3 PZC are changed from $0^{\circ}$ to $45^{\circ}$, respectively. Figures 11 and 12 illustrates FRFs of SS 1 and CC symmetric cross ply $\left(0^{\circ} / 90^{\circ} / 0^{\circ}\right)$ ACLD skew SN Applied Sciences 
Table 5 Fundamental frequency $(\bar{\omega})$ of CC Skew substrate laminates with ACLD of negligible thickness $(\boldsymbol{a}=\boldsymbol{b})$

\begin{tabular}{|c|c|c|c|c|c|c|c|}
\hline \multirow[t]{2}{*}{$\begin{array}{l}\text { Skew } \\
\text { angle }(\alpha)\end{array}$} & \multirow[t]{2}{*}{ Source } & \multicolumn{2}{|c|}{$\begin{array}{l}\text { Symmetric cross-ply } \\
90^{\circ} / 0^{\circ} / 90^{\circ} / 0^{\circ} / 90^{\circ}\end{array}$} & \multicolumn{2}{|c|}{$\begin{array}{l}\text { Antisymmetric cross- } \\
\text { ply } \\
\left(0^{0} / 90^{\circ} / 0^{\circ} / 90^{\circ}\right)\end{array}$} & \multicolumn{2}{|c|}{$\begin{array}{l}\text { Antisymmetric } \\
\text { angle-ply } \\
45^{0} /-45^{0} / 45^{0} /-45^{0}\end{array}$} \\
\hline & & Mode-1 & Mode-2 & Mode-1 & Mode-2 & Mode-1 & Mode-2 \\
\hline \multirow[t]{4}{*}{0} & \multirow[t]{2}{*}{ Ref. [4] } & 2.3820 & 3.7383 & 2.3947 & 3.9533 & 2.2965 & 3.8921 \\
\hline & & 2.3687 & 3.5399 & 2.2990 & 3.7880 & 2.2119 & 3.7339 \\
\hline & Ref. [30] & 2.3201 & 3.4769 & 2.3315 & 3.6531 & 2.2433 & 3.6000 \\
\hline & Present MZZF & 2.3784 & 3.5871 & 2.3933 & 3.7732 & 2.2983 & 3.7120 \\
\hline \multirow[t]{4}{*}{15} & \multirow[t]{2}{*}{ Ref. [4] } & 2.4750 & 3.7872 & 2.4803 & 3.9145 & 2.4007 & 3.8555 \\
\hline & & 2.4663 & 3.6255 & 2.3809 & 3.7516 & 2.3099 & 3.6997 \\
\hline & Ref. [30] & 2.3699 & 3.4821 & 2.3741 & 3.5856 & 2.3049 & 3.5346 \\
\hline & Present MZZF & 2.4305 & 3.5910 & 2.4381 & 3.7024 & 2.3639 & 3.6440 \\
\hline \multirow[t]{4}{*}{30} & \multirow[t]{2}{*}{ Ref. [4] } & 2.7922 & 4.0568 & 2.7798 & 4.1566 & 2.7418 & 4.1221 \\
\hline & & 2.8001 & 3.9557 & 2.6666 & 3.9851 & 2.6325 & 3.9549 \\
\hline & Ref. [30] & 2.5366 & 3.5696 & 2.5240 & 4.1943 & 2.4945 & 3.6113 \\
\hline & Present MZZF & 2.6051 & 3.6809 & 2.5964 & 4.3406 & 2.5653 & 3.7286 \\
\hline \multirow[t]{4}{*}{45} & \multirow[t]{2}{*}{ Ref. [4] } & 3.4739 & 4.7396 & 3.4434 & 4.8223 & 3.4434 & 4.8223 \\
\hline & & 3.5215 & 4.7129 & 3.3015 & 4.6290 & 3.3015 & 4.6290 \\
\hline & Ref. [30] & 2.8665 & 4.7074 & 2.8377 & 4.7614 & 2.8377 & 4.7614 \\
\hline & Present MZZF & 2.9509 & 4.8691 & 2.9294 & 4.9406 & 2.9294 & 4.9406 \\
\hline
\end{tabular}

$\bar{\omega}=\left(\omega b^{2} / \pi^{2} h\right) \sqrt{\rho / E_{2}}, \omega$ is the circular natural frequency.

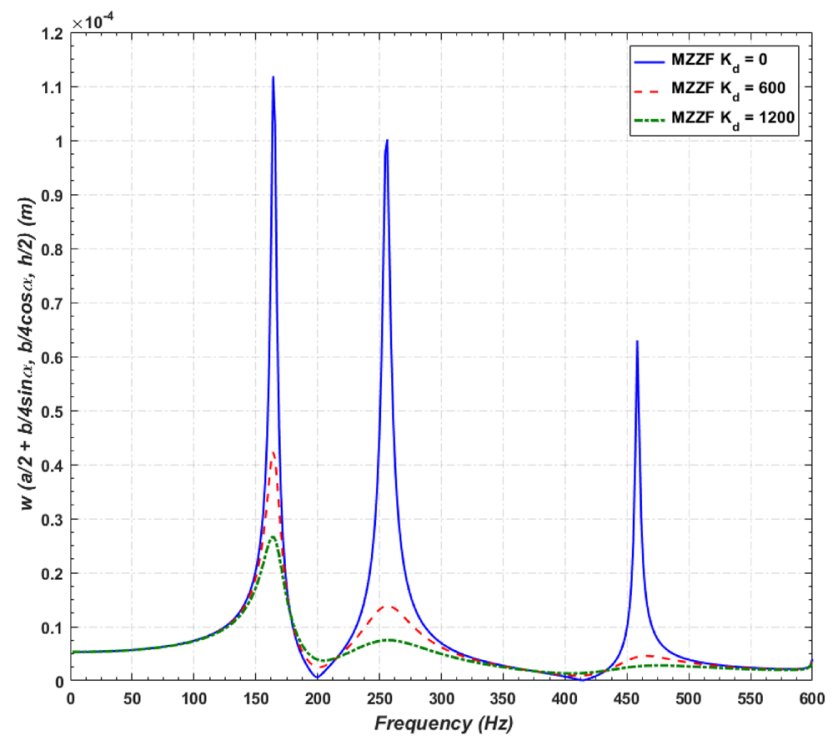

Fig. 4 Frequency response plot of transverse displacement of SS1 ACLD skew plate (Symmetric cross-ply $\left(0^{\circ} / 90^{\circ} / 0^{0}\right), \boldsymbol{\alpha}=15^{\circ}, \mathbf{a} / \mathbf{h}=100, \boldsymbol{\psi}=0^{0}$ )

\section{Conclusion}

The zig-zag behavior in the form of MZZF has been successfully incorporated in the development of FE model of skew plate ACLD system. symmetric cross-ply $\left(0^{\circ} / 90^{\circ} / 0^{\circ}\right)$, antisymmetric cross-ply $\left(0^{\circ} / 90^{\circ} / 0^{\circ} / 90^{\circ}\right)$ and antisymmetric

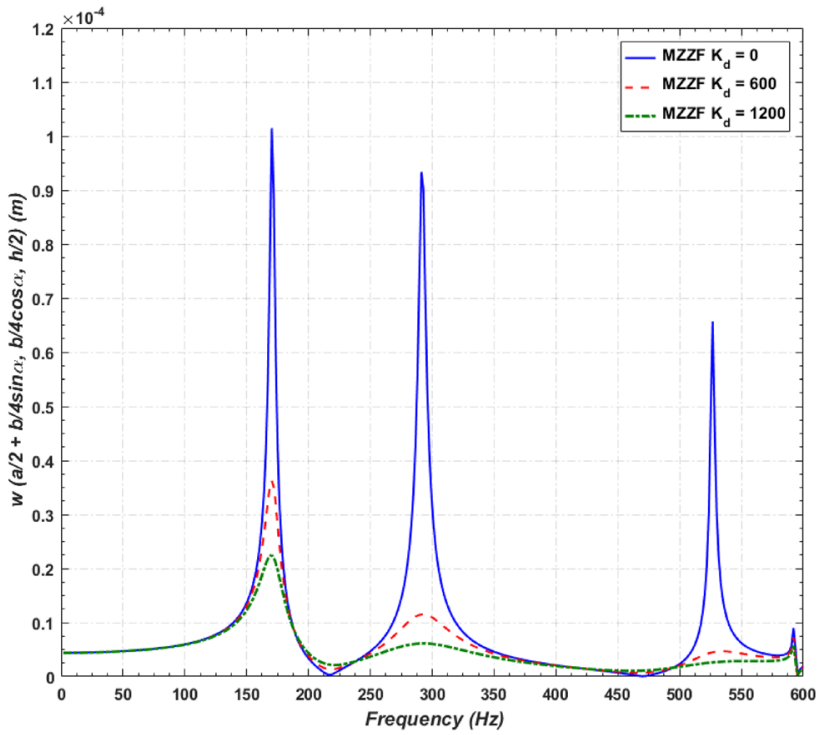

Fig. 5 Frequency response plot of transverse displacement of SS1 ACLD skew plate (Symmetric cross-ply $\left(0^{0} / 90^{\circ} / 0^{0}\right), \boldsymbol{\alpha}=30^{\circ}, \mathbf{a} / \mathbf{h}=100, \boldsymbol{\psi}=0^{0}$ )

angle-ply $\left(45^{\circ} /-45^{\circ} / 45^{\circ} /-45^{\circ}\right)$ substrate skew plates are considered for presenting numerical results. The investigations reveal that the present FE model accurately predicts the damping responses which are consistent with other shear deformation theories. For active damping of the skew plates, he vertical actuation by the 1-3 PZC layers vertical actuation is far better than the in-plane actuation. 


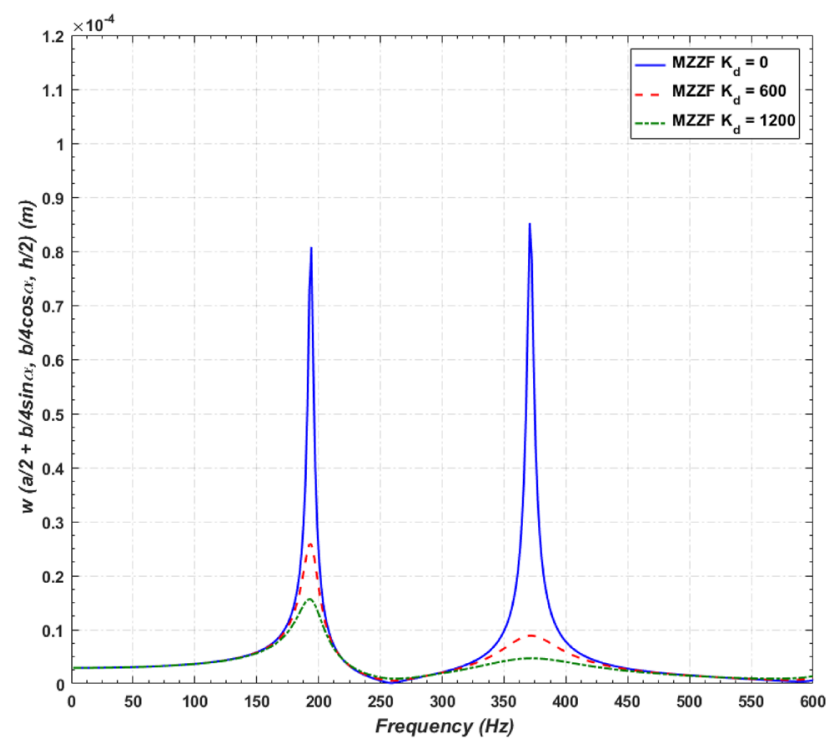

Fig. 6 Frequency response plot of transverse displacement of SS1 ACLD skew plate $\left(\right.$ Symmetric cross-ply $\left.\left(0^{0} / 90^{\circ} / 0^{0}\right), \boldsymbol{\alpha}=45^{0}, \mathbf{a} / \mathbf{h}=100, \boldsymbol{\psi}=0^{0}\right)$

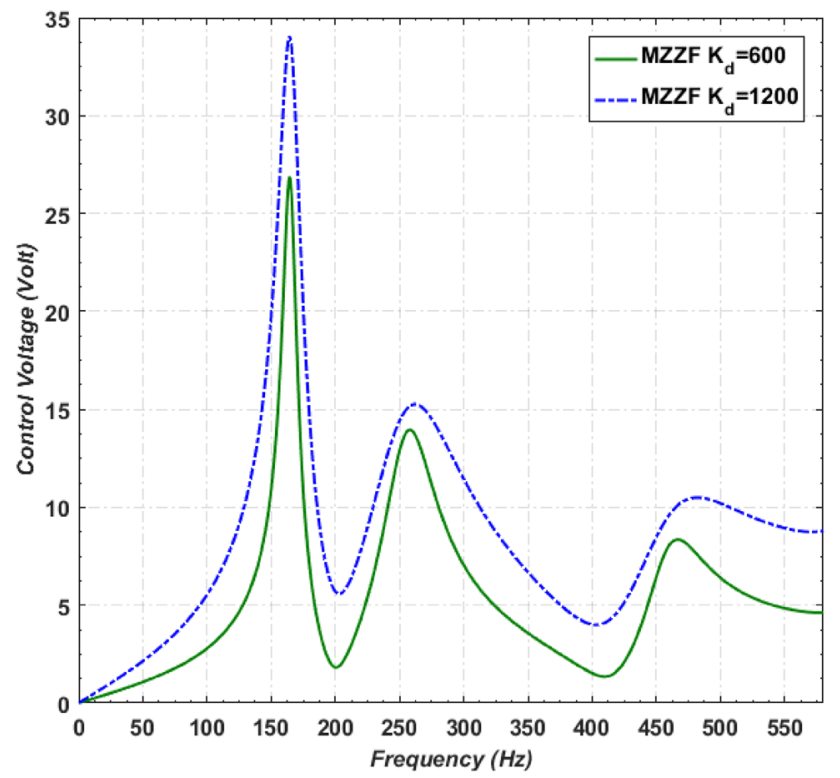

Fig.7 Control voltages for SS1ACLD skew plate $\left(\boldsymbol{\alpha}=15^{0}, \mathbf{a} / \mathbf{h}=100\right)$ (Symmetric cross-ply $\left.\left(0^{\circ} / 90^{\circ} / 0^{\circ}\right), \boldsymbol{\psi}=0^{\circ}\right)$

For the simply-supported end conditions, substrate skew plates with ACLD treatment containing vertically aligned coplanar $\mathbf{x z}$ - and $\mathbf{y z}$-plane piezo fibers $\left(\boldsymbol{\psi}=0^{\circ}\right)$ exhibits better control authority while the same is best when $\boldsymbol{\psi}=$ $30^{\circ}$ for the clamped-clamped end conditions, respectively.

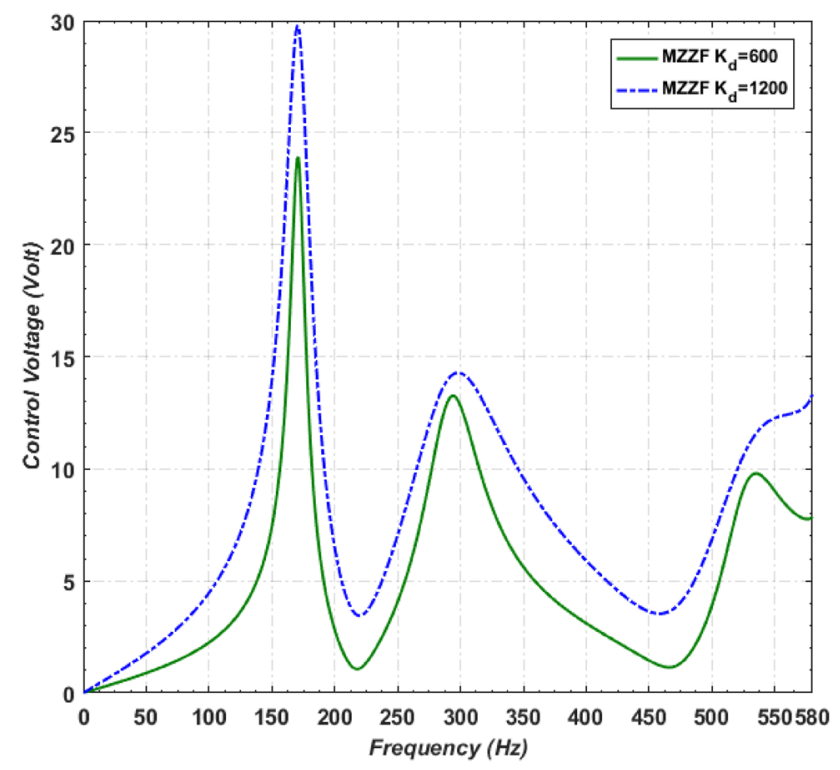

Fig. 8 Control voltages for SS1ACLD skew plate $\left(\boldsymbol{\alpha}=30^{\circ}, \mathbf{a} / \mathbf{h}=100\right)$ (Symmetric cross-ply $\left.\left(0^{\circ} / 90^{\circ} / 0^{\circ}\right), \boldsymbol{\psi}=0^{\circ}\right)$

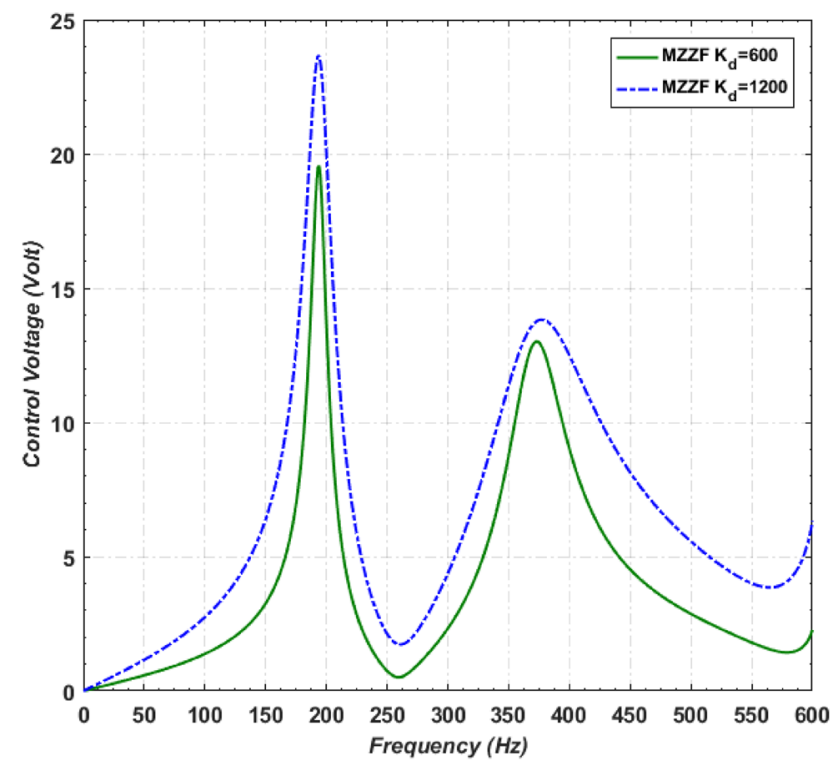

Fig. 9 Control voltages for SS1ACLD skew plate $\left(\boldsymbol{\alpha}=45^{\circ}, \mathbf{a} / \mathbf{h}=100\right)$ (Symmetric cross-ply $\left.\left(0^{\circ} / 90^{\circ} / 0^{\circ}\right), \boldsymbol{\psi}=0^{\circ}\right)$

Limitation of the present study and scope for further research: Although, the study has its own advantages in terms of reduction in time of computation, but the $\mathbf{M Z Z F}$ incorporation for smart control of plates and shells does not give the clear-cut view regarding the across shear 


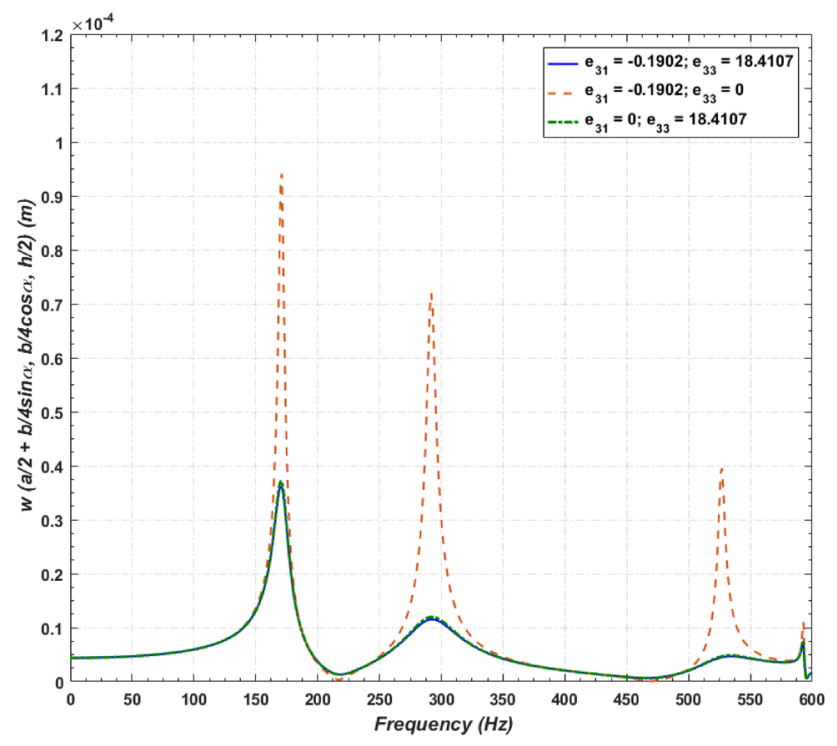

Fig. 10 Significance of piezo-electric coefficient of $e_{33}$ on the frequency response plot of transverse displacement of SS1 ACLD composite plate with control gain $\mathbf{K}_{\mathbf{d}}=1200$ (Symmetric cross-ply $\left(0^{0} / 90^{\circ} / 0^{0}\right), \boldsymbol{\alpha}=45^{\circ}, \mathbf{a} / \mathbf{h}=100, \boldsymbol{\psi}=0^{0}$ )

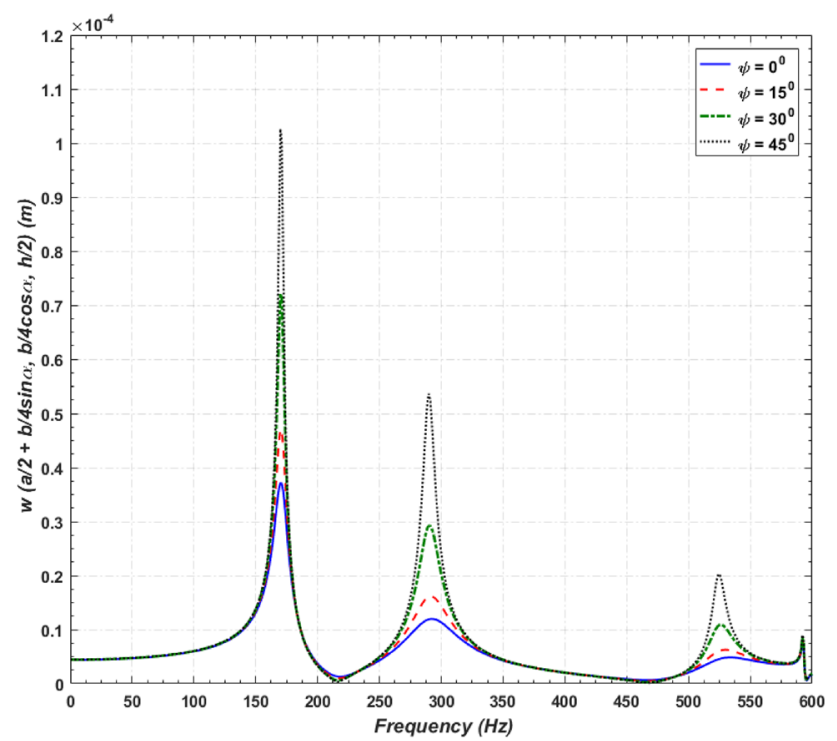

Fig. 11 Effect of piezo-fiber angle ( $\boldsymbol{\psi}$ coplanar with $\mathbf{x z}$-plane) on the control aspect of the Patches in damping the responses of SS1 skew substrate ACLD, (Symmetric cross-ply $\left.\left(0^{0} / 90^{\circ} / 0^{0}\right), \boldsymbol{\alpha}=30^{\circ}, \mathbf{K}_{\mathbf{d}}=1200\right)$

which is close to parabolic nature. This may be sorted out by including the MZZF in the displacement fields of HSDT providing ample scope for further research.

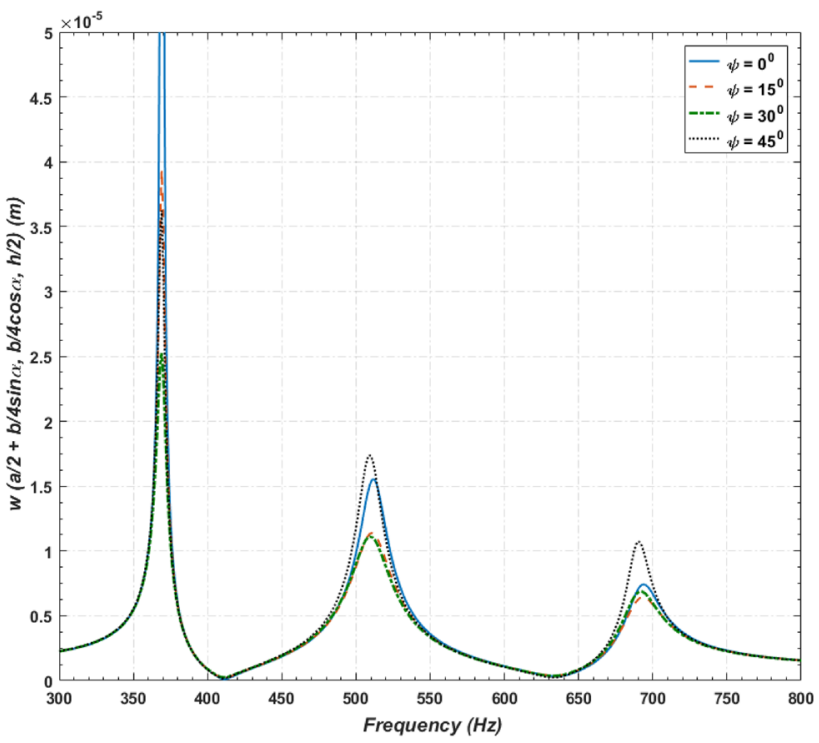

Fig. 12 Effect of piezo-fiber angle ( $\boldsymbol{\psi}$ coplanar with $\mathbf{x z}$-plane) on the control aspect of the Patches in damping the responses of CC skew substrate ACLD, (Symmetric cross-ply $\left(0^{0} / 90^{\circ} / 0^{0}\right), \boldsymbol{\alpha}=30^{\circ}, \mathbf{K}_{\mathbf{d}}=1200$ )

\section{Compliance with ethical standards}

Conflict of interest The authors declare that they do not have conflict of interest any in the preparation of the article.

Open Access This article is licensed under a Creative Commons Attribution 4.0 International License, which permits use, sharing, adaptation, distribution and reproduction in any medium or format, as long as you give appropriate credit to the original author(s) and the source, provide a link to the Creative Commons licence, and indicate if changes were made. The images or other third party material in this article are included in the article's Creative Commons licence, unless indicated otherwise in a credit line to the material. If material is not included in the article's Creative Commons licence and your intended use is not permitted by statutory regulation or exceeds the permitted use, you will need to obtain permission directly from the copyright holder. To view a copy of this licence, visit http://creativecommons. org/licenses/by/4.0/.

\section{References}

1. Wang S (1997) Free vibration analysis of skew fibre-reinforced composite laminates based on first-order shear deformation plate theory. Comput Struct 63:525-538

2. Krishna Reddy AR, Palaninathan R (1999) Free vibration of skew laminates. Compos Struct 70:415-423

3. Sarath Babu C, Kant T (1999) Two shear deformable finite element models for buckling analysis of skew fibre-reinforced composite and sandwich panels. Compos Struct 46:115-124 
4. Garg AK, Khare RK, Kant T (2006) Free vibration of skew fiberreinforced composite and sandwich laminates using a shear deformable finite element model. J Sandw Struct Mater 8:33-53

5. Kiani $Y$ (2017) Thermal buckling of temperature dependent FG-CNT reinforced composite skew plates. J Therm Stress 40:1442-1460

6. Kallannavar V, Kumaran B, Kattimani SC (2019) Effect of temperature and moisture on free vibration characteristics of skew laminated hybrid composite and sandwich plates. Thin Walled Struct 157:107113

7. Murakami H (1986) Laminated composite plate theory with improved in-plane responses. J Appl Mech 53:661-666

8. Carrera E (2004) On the use of the Murakami's zig-zag function in the modeling of layered plates and shells. Compu Struct 82:541-554

9. Carrera E, Ciuffreda A (2005) A unified formulation to assess theories of multilayered plates for various bending problems. Compos Struct 69:271-293

10. Brischetto S, Carrera E, Demasi L (2009) Improved response of unsymmetrically laminated sandwich plates by using zig-zag functions. J Sandw Struct Mater 11:257-267

11. Brischetto S, Carrera E, Demasi L (2009) Improved bending analysis of sandwich plates using a zig-zag function. Compos Struct 89:408-415

12. Bailey T, Hubbard JE (1985) Distributed piezoelectric-polymer active vibration control of a cantilever beam. J Guid Control Dyn 8:605-611

13. Reddy JN (1999) On laminated composite plates with integrated sensors and actuators. Eng Struct 21:568-593

14. Elahi H, Eugeni M, Gaudenzi P, Qayyum F, Swati RF, Khan HM (2018) Reponse of piezoelectric materials on thermomechanical shocking and electrical shocking for aerospace applications. Micro Syst Technol 24:3791-3798

15. Smith WA, Auld BA (1991) Modeling 1-3 Composite Piezoelectrics: Thickness-Mode Oscillations. IEEE Trans Ultrason Ferroelectr Freq Control 38:40-47

16. Dunn ML, Taya M (1993) Micromechanics predictions of the effective electroelastic moduli of piezoelectric composites. Int J Solids Struct 30:161-175

17. Stevenson T, Martin DG, Cowin PI, Blumfield A, Bell AJ, Comyn TP, Weaver PM (2015) Piezoelectric materials for high temperature transducers and actuators. J Mater Sci Mater Electron 26:9256-9267

18. Wang S, Rong W, Wang L, Xie H, Sun L, Mills JK (2019) A survey of piezoelectric actuators with long working stroke in recent years: classification, principles, connections and distinction. Mech Syst Signal Process 123:591-605

19. Piezocomposites, Materials Systems Inc., 543 Great Road, Littleton, MA 01460

20. Baz A (1996) Active constrained layer damping. U.S. Patent $5,485,053$

21. Ray MC, Baz A (1997) Optimization of energy dissipation of active constrained layer damping treatments of plates. J Sound Vib 208:391-406

22. Ray MC, Pradhan AK (2007) On the use of vertically reinforced 1-3 piezoelectric composites for hybrid damping of laminated composite plates. Mech Adv Mater Struct 14:245-261

23. Kanasogi RM, Ray MC (2013) Active constrained layer damping of smart skew laminated composite plates using 1-3 piezoelectric composites. J Compos 2013:1-17

24. Selim BA, Zhang LW, Liew KM (2016) Active vibration control of FGM plates with piezoelectric layers based on Reddy's higherorder shear deformation theory. Compos Struct 155:118-134

25. Zhao J, Wong PK, Ma X, Xie ZC, Xu J, Cristino VA (2019) Simplification of finite element modeling for plates structures with constrained layer damping treatment by using single-layer equivalent material properties. Compos Part B 157:283-288

26. Selim BA, Liu Z, Liew KM (2019) Active vibration control of functionally graded graphene nanoplatelets reinforced composite plates integrated with piezoelectric layers. Thin Walled Struct. https://doi.org/10.1016/j.tws.2019.106372

27. Li J, Xue Y, Li F, Narita Y (2019) Active vibration control of functionally graded piezoelectric material plate. Compos Struct 207:509-518

28. Vinyas M (2019) Vibration control of skew magneto-electro elastic plates using active constrained layer damping. Compos Struct 208:600-616

29. Khan NM, Kumar RS (2021) Smart control of cylindrical shells incorporating Murakami zig-zag function. Compos Struct 257:113044

30. Reddy JN (1996) Mechanics of laminated composites plates theory and analysis. CRC Press, Boca Raton

Publisher's Note Springer Nature remains neutral with regard to jurisdictional claims in published maps and institutional affiliations. 